\title{
Five-dimensional gauge theories on spheres with negative couplings
}

\author{
Joseph A. Minahan ${ }^{a}$ and Anton Nedelin ${ }^{b}$ \\ ${ }^{a}$ Department of Physics and Astronomy, Uppsala university, \\ Box 516, SE-75120 Uppsala, Sweden \\ ${ }^{b}$ Deparment of Physics, Technion, \\ 32000 Haifa, Israel \\ E-mail: joseph.minahan@physics.uu.se, anton.nedelin@physics.uu.se
}

ABStract: We consider supersymmetric gauge theories on $S^{5}$ with a negative Yang-Mills coupling in their large $N$ limits. Using localization we compute the partition functions and show that the pure $\mathrm{SU}(N)$ gauge theory descends to an $\mathrm{SU}(N / 2)_{+N / 2} \times \mathrm{SU}(N / 2)_{-N / 2} \times$ $\mathrm{SU}(2)$ Chern-Simons gauge theory as the inverse 't Hooft coupling is taken to negative infinity for $N$ even. The Yang-Mills coupling of the $\mathrm{SU}(N / 2)_{ \pm N / 2}$ is positive and infinite, while that on the $\mathrm{SU}(2)$ goes to zero. We also show that the odd $N$ case has somewhat different behavior. We then study the $\mathrm{SU}(N / 2)_{N / 2}$ pure Chern-Simons theory. While the eigenvalue density is only found numerically, we show that its width equals 1 in units of the inverse sphere radius, which allows us to find the leading correction to the free energy when turning on the Yang-Mills term. We then consider $\operatorname{USp}(2 N)$ theories with an antisymmetric hypermultiplet and $N_{f}<8$ fundamental hypermultiplets and carry out a similar analysis. Along the way we show that the one-instanton contribution to the partition function remains exponentially suppressed at negative coupling for the $\operatorname{SU}(N)$ theories in the large $N$ limit.

Keywords: 1/N Expansion, Matrix Models, Supersymmetric Gauge Theory

ARXiv EPrint: 2007.13760 


\section{Contents}

1 Introduction 1

2 The partition function on $S^{5}$ with an adjoint hypermultiplet $\quad 6$

$\begin{array}{lll}3 & \mathrm{SU}(N) \text { gauge theory with negative coupling } & 8\end{array}$

$\begin{array}{ll}3.1 & N \text { even }\end{array}$

$3.2 \quad N$ odd 11

$\begin{array}{ll}3.3 & \text { The Chern-Simons levels directly from field theory } \\ \end{array}$

$4 \mathrm{SU}(N / 2)_{N / 2}$ Chern-Simons $\quad 14$

5 Instantons at large $N$ in 5D $\quad 16$

$6 \quad \operatorname{USp}(2 N)$ gauge theories $\quad 18$

$\begin{array}{lll}\text { 6.1 Negative } \lambda_{\text {eff }} \text { with decoupled fundamental hypermultiplets } & 19\end{array}$

6.2 Negative $\lambda_{\text {eff }}$ with a decoupled antisymmetric hypermultiplet 21

6.3 An apparent fifth order phase transition and its possible resolution 23

$\begin{array}{llr}7 & \text { Discussion and outlook } & 26\end{array}$

$\begin{array}{ll}\text { A Width of the eigenvalue distribution at finite } N & 27\end{array}$

B Exponential behavior for instantons $\quad 28$

\section{Introduction}

Negative couplings in supersymmetric gauge theories can have a perfectly well-defined interpretation. One well known example is pure $\mathcal{N}=1 \mathrm{SU}(2)$ gauge theory in five dimensions which has a real one-dimensional Coulomb branch [1]. At a generic value of the coupling there is a topological $\mathrm{U}(1)$ global symmetry whose current is $j=\frac{1}{32 \pi^{2}} *(F \wedge F)$. The objects charged under this symmetry are the instantons, which in five dimensions are particles with mass given by $m_{I}=\frac{4 \pi^{2}}{g_{\mathrm{YM}}^{2}}$. On the Coulomb branch the instantons are also charged under the unbroken U(1) gauge symmetry which shifts the mass by the scalar expectation value $\phi$.

At small positive coupling the instantons are very heavy, but become massless in the UV at infinite coupling when sitting at the origin of the Coulomb branch. At this point the theory is superconformal and the global $\mathrm{U}(1)$ symmetry is enhanced to $\mathrm{SU}(2)$ [1]. At this $\mathrm{SU}(2)$ point one can implement a Weyl transformation that flips the direction of the coupling, such that moving back down in the coupling moves it to the negative side. This 


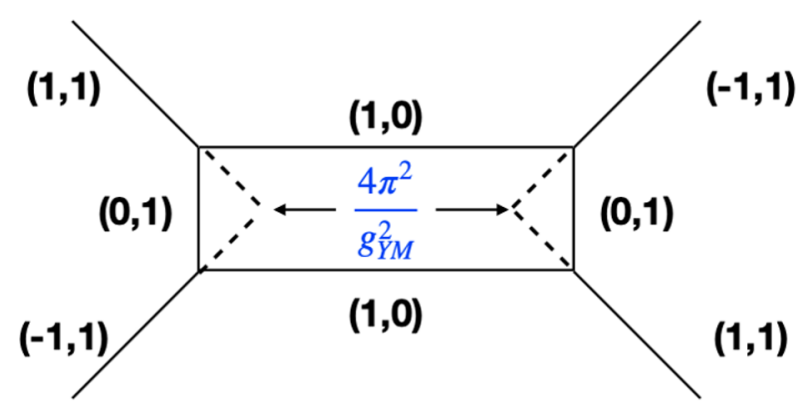

(a) $g_{\mathrm{YM}}^{2}>0$

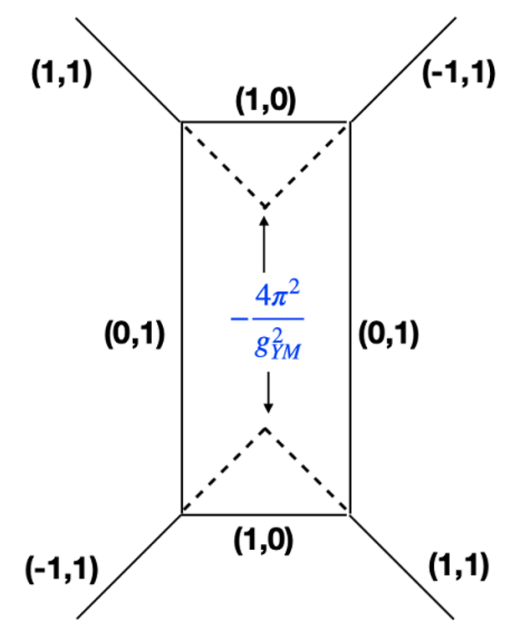

(b) $g_{\mathrm{YM}}^{2}<0$

Figure 1. $(p, q)$ web for $\mathcal{N}=1 \mathrm{SU}(2)$ gauge theory at positive and negative coupling. D5 branes are $(1,0)$ branes and NS5 branes are $(0,1)$. The coupling is determined by the positions of the fixed $( \pm 1,1)$ external branes.

transformation also switches the instantons with the original $W$-bosons, such that the $W$ bosons have a mass $\phi-\frac{4 \pi^{2}}{g_{\mathrm{YM}}^{2}}$, while the instantons now have mass $\phi$. At the origin of this new Coulomb branch it is now the instantons that enhance the gauge symmetry to $\mathrm{SU}(2)$.

This story has a nice description in terms of $(p, q) 5$ branes as shown in figure $1[2,3]$. The instantons are D1 branes that stretch between the two NS5 branes, separated by a distance $\frac{4 \pi^{2}}{g_{\mathrm{YM}}^{2}}$, while the $W$-bosons are $F$-strings that attach to the two D5 branes. As we increase the coupling the NS5 branes move closer together. Moving through the fixed point the roles of the NS5 branes and the D5 branes are exchanged.

To go to higher rank $\mathrm{SU}(N)$ gauge groups we add D5 branes to the diagram in figure 1, as shown in figure 2 . In this case one can still pass through to negative coupling, but there is no longer a symmetry between the positive and negative sides. However, there is still interesting behavior. In particular, if we assume that $N$ is even we see that at the origin of the Coulomb branch the branes split into two groups of $N / 2$, separated by $-\frac{8 \pi^{2}}{\lambda}$ where 


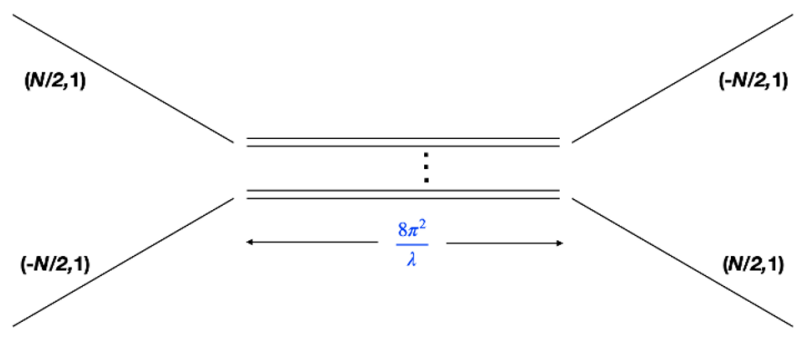

(a) $g_{\mathrm{YM}}^{2}>0$

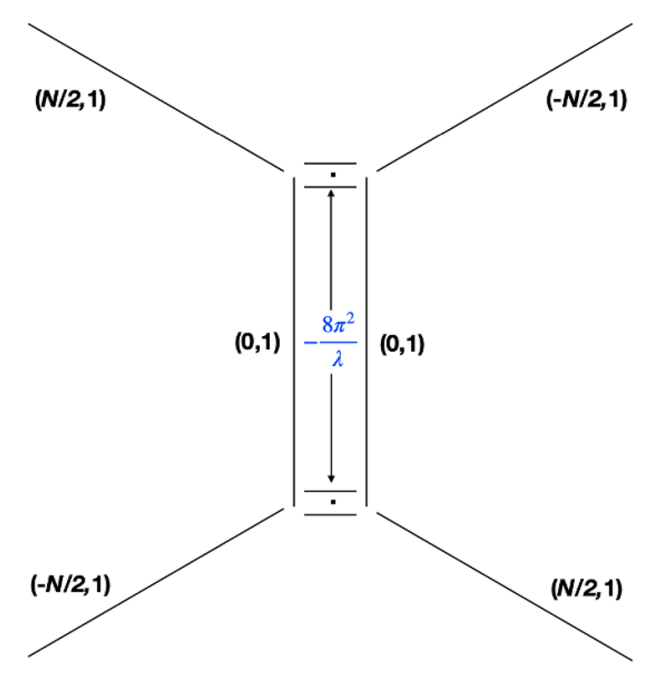

(b) $g_{\mathrm{YM}}^{2}<0$

Figure 2. $(p, q)$ web for $\mathcal{N}=1 \mathrm{SU}(N)$ gauge theory at positive and negative coupling with $N$ even. The webs represent the origin of the Coulomb branch. At negative coupling the D5 branes split into two groups of $N / 2$.

$\lambda=g_{\mathrm{YM}}^{2} N$ is the 't Hooft coupling. In the limit that $-\frac{4 \pi^{2}}{\lambda}$ approaches infinity, the two sets of D5 branes move far apart from each other, with each half described by the web in figure 3, or its vertical reflection. This is the $(p, q)$ web for an $\mathrm{SU}(N / 2)$ gauge theory with a five-dimensional Chern-Simons term at level $k=N / 2$, while the vertical reflected web is at level $k=-N / 2$. Both gauge theories have infinite Yang-Mills coupling. Hence, the resulting theory is $\mathrm{SU}(N / 2)_{N / 2} \times \mathrm{SU}(N / 2)_{-N / 2} \times \mathrm{SU}(2)$, where the $\mathrm{SU}(2)$ comes from the parallel NS5 branes and is weakly coupled.

If we put the theory on $S^{5}$ we can use localization $[4,5]$ to compute the free energy and certain supersymmetric observables by reducing the theory to a matrix model. If we assume that we are in the large $N$ limit then these quantities can be evaluated by saddle point. For this setup it should be possible to pass through the infinite coupling point to negative coupling and examine the behavior. While one might expect the negative coupling to destabilize the matrix model, the one-loop determinant more than compensates for the negative coupling and renders the entire matrix model stable. We will see that at negative coupling the localized path integral is dominated by a saddle point where the $N$ eigenvalues 


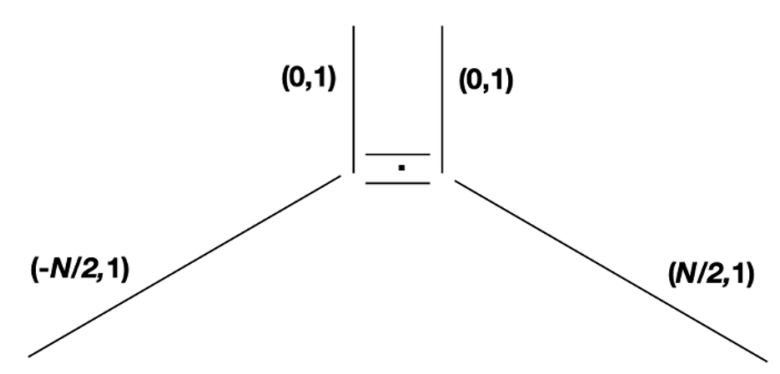

Figure 3. $(p, q)$ web for $\mathcal{N}=1 \mathrm{SU}(N / 2)$ gauge theory with a Chern-Simons term at level $k=N / 2$.

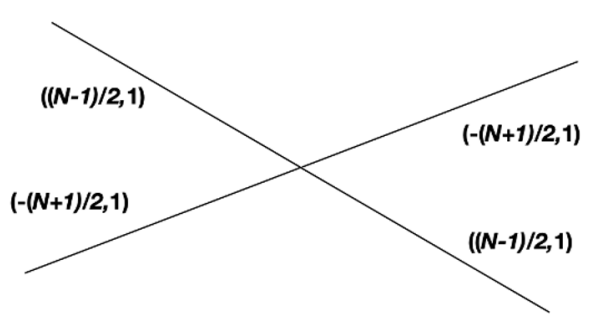

(a) $g_{\mathrm{YM}}^{2}=\infty$

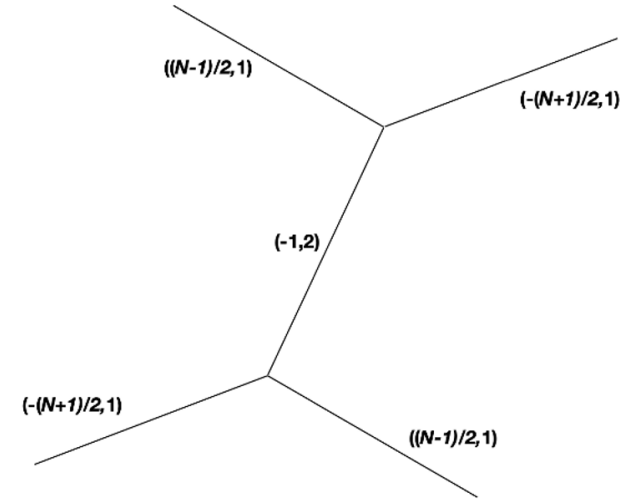

(b) $g_{\mathrm{YM}}^{2}<0$

Figure 4. $(p, q)$ web for $\mathcal{N}=1 \mathrm{SU}(N)$ gauge theory with $N$ odd.

split into two groups where the mean position of the two groups is separated by $-\frac{8 \pi^{2}}{\lambda}$. Within each group, the eigenvalues take the distribution one would get for an $\mathrm{SU}(N / 2)$ Chern-Simons theory at level $\pm N / 2$. There is also a $\mathrm{U}(1)$ gauge theory with a positive weakly coupled Yang-Mills term which is enhanced to SU(2) by massless instantons.

For odd $N$ the result on $S^{5}$ is somewhat subtle. At infinite coupling and at the origin of the Coulomb branch the $(p, q)$ web has the configuration shown in figure 4(a). Passing through the fixed point to negative coupling a $(-1,2)$ brane joins the two sets of external branes as shown in figure 4(b). One can then move onto the Coulomb branch with the appearance of hidden faces [3], as shown in figure 5(a). Here one has two sets of separated $(N-1) / 2$ D5 branes along with a half D5 brane at the tip of each triangle. As the faces get larger the tips will eventually merge to form a D5 brane at the origin as shown in figure 5(b).

On the $S^{5}$ one integrates the $N-1$ eigenvalues over the Coulomb branch, thus it seems that the relevant web is the one in figure 5(b). Here we will find that the saddle point at negative coupling will be dominated by two sets of $(N-1) / 2$ eigenvalues far apart from each other, with one more eigenvalue at the origin, consistent with the picture in figure $5(\mathrm{~b})$. The two sets of eigenvalues approach the profiles of an $\mathrm{SU}\left(\frac{N-1}{2}\right)$ gauge theory with levels $k= \pm(N+1) / 2$. The two gauge theories also have induced Yang-Mills actions with positive coupling, whose inverse is equal to half of the length of the D5 branes pictured 
in figure 5(b). Hence their couplings go to zero as the 't Hooft coupling of the original $\mathrm{SU}(N)$ gauge theory approaches $0_{-}$.

We can also consider each $\mathrm{SU}(N / 2)_{ \pm N / 2}$ Chern-Simons as a stand-alone theory. At infinite Yang-Mills coupling each is superconformal. On the $S^{5}$ we can find the eigenvalue density of the corresponding matrix model numerically. While we have not succeeded in finding the distribution analytically, we can show that the width of the distribution is exactly 1 in the large $N$ limit in units of the inverse sphere radius. We can also show that the distribution has a tail in one direction that falls off exponentially with a rate that can also be computed analytically. The infinite tail suggests that only a positive Yang-Mills term can be turned on to move away from the fixed point. Using the known width of the distribution we can find the leading order correction to the free energy coming from the Yang-Mills term.

As in four dimensions [6], one might worry that the negative gauge coupling could lead to an enhancement of the instanton contribution in the partition function. The instantons are localized on the vertices of the toric base of the $C P^{2}$, which itself is the base of a circle fibration for the $S^{5}$ [7-9]. The contribution of the instantons at each vertex on the base are divergent in the limit of a round sphere. However, by turning on small squashing parameters we can show that the divergence cancels when adding up the contribution from all vertices. After canceling the divergences one can turn off the squashing parameters and evaluate the resulting one-instanton contribution numerically. We can then show that this expression remains exponentially suppressed in $N$ when the coupling is negative, demonstrating that the instanton contribution to the partition function can be safely ignored.

We can also consider $\operatorname{USp}(2 N)$ gauge theories. If the theory has a massless hypermultiplet in the antisymmetric representation as well as $N_{f}<8$ massless hypermultiplets in the fundamental representation, then it has a superconformal fixed point at infinite YangMills coupling that is dual to a weakly coupled supergravity theory on $A d S_{6}$ [10-12]. Here we can also consider turning on a negative Yang-Mills coupling, where one finds that the eigenvalue distribution splits into a peak and its reflection. The behavior is similar to the pure $\mathrm{SU}(N)$ case with $N$ odd. We then investigate the behavior of the $\operatorname{USp}(2 N)$ theory near the fixed point. Unlike the pure $\mathrm{SU}(N)$ case, this theory seems to exhibit a phase transition as one passes from positive to negative inverse coupling. We show that this apparent phase transition is fifth order. However this result contradicts the self-duality of the $\operatorname{USp}(2 N)$ that follows from the $\mathrm{SU}(2)$ global symmetry of the corresponding fixed point. We propose that a full accounting of instantons will resolve this contradiction, but leave its proof for the future.

The rest of the paper is organized as follows. In section 2 we review the matrix model derived from an $\mathrm{SU}(N)$ gauge theory with an adjoint hypermultiplet of mass $m$. By taking the mass to infinity we reduce the theory to a pure $\mathrm{SU}(N)$ theory with an effective 't Hooft coupling that can be tuned to be negative. In section 3 we study this theory at negative coupling for even and odd $N$ and compare the behavior to our expectations from the $(p, q)$ webs. We also show how one generates the Chern-Simons levels of the resulting theories by integrating out the massive fermions. In section 4 we consider an $\mathrm{SU}(N / 2)$ gauge theory at Chern-Simons level $N / 2$ on the $S^{5}$. We find the eigenvalue density numerically 


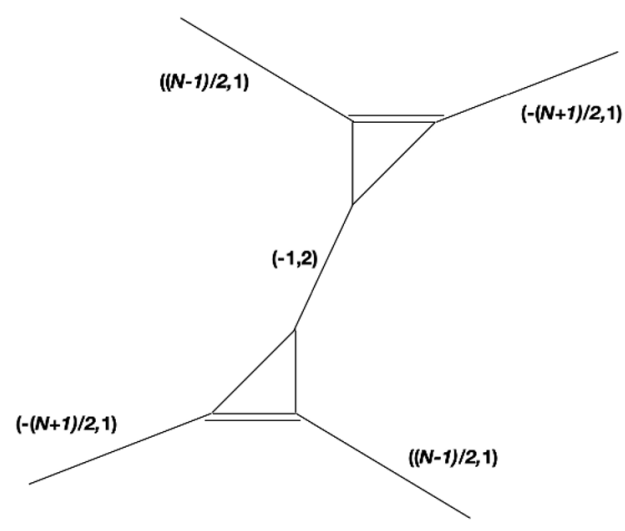

(a) $g_{\mathrm{YM}}^{2}<0$ along the Coulomb branch

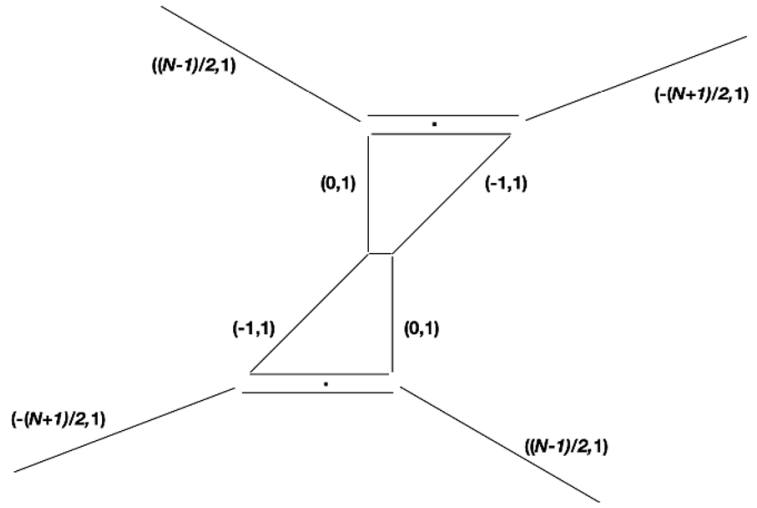

(b) $g_{\mathrm{YM}}^{2}<0$ with D5 brane at the origin

Figure 5. $(p, q)$ webs for $\mathcal{N}=1 \mathrm{SU}(N)$ gauge theory with $N$ odd at two points along the Coulomb branch from figure 4 . (a) shows the hidden faces which merge to form a D5 brane in (b).

and show that it has an exponential tail that extends to infinity. We find the width of the distribution analytically and use this to find the correction to the free energy by turning on a Yang-Mills term. In section 5 we compute the one-instanton contribution to the partition function numerically and show that it is exponentially suppressed in $N$. In section 6 we consider the $\operatorname{USp}(2 N)$ theories. We first study these theories at negative coupling. We then consider its behavior near the fixed point and show how the matrix model leads to a fifth order phase transition. In section 7 we present our conclusions. The appendices contain extra technical details.

\section{The partition function on $S^{5}$ with an adjoint hypermultiplet}

In this section we consider the matrix model for $\mathrm{SU}(N) \mathcal{N}=1$ super Yang-Mills (SYM) with an adjoint hypermultiplet with mass $m$ on $S^{5}$. The hypermultiplet mass will be taken to infinity such that it reduces to pure $\mathcal{N}=1 \mathrm{SYM}$ with a negative effective coupling.

The localized partition function for an $\mathrm{SU}(N)$ gauge theory with eight supersymmetries on $S^{d}$ has the general form $[13,14]^{1}$

$$
Z=\int_{\text {Cartan }}[d \sigma] e^{-\frac{4 \pi \frac{d+1}{2} r d-4}{g_{\mathrm{YM}}^{2} \Gamma\left(\frac{d-3}{2}\right)} \operatorname{Tr} \sigma^{2}} Z_{1-\text { loop }}(\sigma)+\text { Instantons }
$$

where $\sigma$ is an $N \times N$ Hermitian matrix. ${ }^{2} Z_{1 \text {-loop }}(\sigma)$ is the contribution of the Gaussian fluctuations about the localized fixed point. Its contribution from the vector multiplet, combined with the Vandermonde determinant is given by

$$
Z_{1-\text { loop }}^{\text {vect }}(\sigma) \prod_{\beta>0}\langle\beta, \sigma\rangle^{2}=\prod_{\beta>0} \prod_{n=0}^{\infty}\left(\left(n^{2}+\langle\beta, \sigma\rangle^{2}\right)\left((n+d-2)^{2}+\langle\beta, \sigma\rangle^{2}\right)\right)^{\frac{\Gamma(n+d-2)}{\Gamma(n+1) \Gamma(d-2)}} .
$$

\footnotetext{
${ }^{1}$ For particular expressions in $5 d$ case see [15-17].

${ }^{2}$ We use the conventions for $g_{\mathrm{YM}}^{2}$ in [18].
} 
where $\gamma$ are the positive roots for the gauge group. Likewise, the contribution from the adjoint hypermultiplet is

$$
Z_{1 \text {-loop }}^{\text {hyper }}(\sigma)=\prod_{\beta} \prod_{n=0}^{\infty}\left[\left(\left(n+\frac{d-2}{2}\right)^{2}+(\langle\beta, \sigma\rangle+\mu)^{2}\right)\right]^{-\frac{\Gamma(n+d-2)}{\Gamma(n+1) \Gamma(d-2)}}
$$

where $\mu \equiv m r$ is the dimensionless mass parameter.

In the large- $N$ limit we can ignore the contribution of instantons (to be shown explicitly in section 5) and the partition function is dominated by a saddle point. If we set $d=5$ we find the saddle point equations [19]

$$
\begin{aligned}
\frac{8 \pi^{3} N}{\lambda} \sigma_{i}=\pi \sum_{j \neq i}[ & \left(2-\left(\sigma_{i}-\sigma_{j}\right)^{2}\right) \operatorname{coth}\left(\pi\left(\sigma_{i}-\sigma_{j}\right)\right) \\
& +\frac{1}{2}\left(\frac{1}{4}+\left(\sigma_{i}-\sigma_{j}-\mu\right)^{2}\right) \tanh \left(\pi\left(\sigma_{i}-\sigma_{j}-\mu\right)\right) \\
& \left.+\frac{1}{2}\left(\frac{1}{4}+\left(\sigma_{i}-\sigma_{j}+\mu\right)^{2}\right) \tanh \left(\pi\left(\sigma_{i}-\sigma_{j}+\mu\right)\right)\right],
\end{aligned}
$$

where $\lambda \equiv g_{\mathrm{YM}}^{2} N / r$ is the dimensionless 't Hooft coupling.

Let us now assume that the mass parameter $\mu$ is very large, such that $\mu \gg 1$ and $\mu \gg\left|\sigma_{i}-\sigma_{j}\right|$ for all $i$ and $j$. In this case the hypermultiplet mass acts as a regulator and the saddle point equations reduces to

$$
\frac{8 \pi^{3} N}{\lambda_{\text {eff }}} \sigma_{i}=\pi \sum_{j \neq i}\left(2-\left(\sigma_{i}-\sigma_{j}\right)^{2}\right) \operatorname{coth}\left(\pi\left(\sigma_{i}-\sigma_{j}\right)\right)
$$

where $\lambda_{\text {eff }}$ is the effective 't Hooft coupling which is defined by

$$
\frac{4 \pi^{2}}{\lambda_{\mathrm{eff}}} \equiv\left(\frac{4 \pi^{2}}{\lambda}-\mu\right)
$$

Equation (2.5) is the saddle point equation for a vector multiplet and in deriving it we used that $\sum_{i} \sigma_{i}=0$ since the gauge group is $\mathrm{SU}(N)$ and not $\mathrm{U}(N)$.

For small separations where $\left|\sigma_{i j}\right| \ll 1$, the kernel in (2.5) behaves as

$$
\pi\left(2-\left(\sigma_{i}-\sigma_{j}\right)^{2}\right) \operatorname{coth}\left(\pi\left(\sigma_{i}-\sigma_{j}\right)\right) \approx \frac{2}{\sigma_{i j}} .
$$

This is relevant at weak effective coupling when $\lambda_{\text {eff }}^{-1} \gg 1$. However, for large separations the kernel behaves as

$$
\pi\left(2-\left(\sigma_{i}-\sigma_{j}\right)^{2}\right) \operatorname{coth}\left(\pi\left(\sigma_{i}-\sigma_{j}\right)\right) \approx-\pi\left(\sigma_{i}-\sigma_{j}\right)^{2} \operatorname{sign}\left(\sigma_{i}-\sigma_{j}\right),
$$

which is half the $7 \mathrm{~d}$ large separation kernel with 16 supersymmetries [18]. Hence we expect the eigenvalues to behave similarly to that case. Indeed one finds with the approximation in (2.8) that the eigenvalue density, defined as

$$
\rho(\sigma) \equiv N^{-1} \sum_{i=1}^{N} \delta\left(\sigma-\sigma_{i}\right)
$$


reduces to $[18,20]$

$$
\rho(\sigma)=\frac{1}{2}(\delta(\sigma+b)+\delta(\sigma-b))
$$

where $b=-\frac{4 \pi^{2}}{\lambda_{\text {eff }}}$. Hence the approximation in (2.8) is valid if $\lambda_{\text {eff }}^{-1} \ll-1$. In addition, we can borrow from the $7 \mathrm{~d}$ result in [18] to show that here the free energy in this limit is

$$
\begin{aligned}
F & =N^{2}\left(\frac{4 \pi^{2}}{\lambda_{\text {eff }}} \int_{-b}^{b} d \sigma \rho(\sigma) \sigma^{2}+\frac{\pi}{6} \int_{-b}^{b} d \sigma \rho(\sigma) \int_{-b}^{b} d \sigma^{\prime} \rho\left(\sigma^{\prime}\right)\left|\sigma-\sigma^{\prime}\right|^{3}\right) \\
& =\frac{4 \pi^{3}}{3 \lambda_{\text {eff }}} N^{2}\left(-\frac{4 \pi^{2}}{\lambda_{\text {eff }}}\right)^{2}=\frac{64}{3} \frac{\pi^{7}}{\lambda_{\text {eff }}^{3}} N^{2}
\end{aligned}
$$

We may also consider BPS Wilson loops that wrap the $S^{5}$ equator. These have the expectation value

$$
\langle W\rangle=\operatorname{Tr}\left(\mathbf{P} e^{i \oint d s \cdot \phi_{0}}\right) \approx \int_{-b}^{b} d \sigma \rho(\sigma) e^{2 \pi \sigma}
$$

Using the eigenvalue density in (2.10) we find

$$
\langle W\rangle=\cosh \left(\frac{8 \pi^{3}}{\lambda_{\text {eff }}}\right) \approx \frac{1}{2} \exp \left(-\frac{8 \pi^{3}}{\lambda_{\text {eff }}}\right)
$$

\section{$3 \quad \mathrm{SU}(N)$ gauge theory with negative coupling}

In this section we consider more closely the behavior of the eigenvalue density when $\lambda_{\text {eff }}^{-1} \ll-1$ and how it meshes with our understanding from the $(p, q)$ webs. Note that we can naturally approach this regime when flowing to the IR. To see this, let us make the radius of the $S^{5}$ explicit in (2.6), so that we have

$$
\frac{4 \pi^{2}}{\lambda_{\mathrm{eff}}}=\frac{r}{N}\left(\frac{4 \pi^{2}}{g_{\mathrm{YM}}^{2}}-m N\right) .
$$

The hypermultiplet mass $m$ and the bare coupling $g_{\mathrm{YM}}^{2}$ may be considered fixed. We can then flow to the UV by sending $r \rightarrow 0$. In this case $\lambda_{\text {eff }}^{-1} \rightarrow 0$, independent of the sign, and we reach a nontrivial UV fixed point $[1,21]$.

However, if we send $r \rightarrow \infty$ such that we flow to the IR, then the sign matters. If $\lambda_{\text {eff }}>0$ then the flow is to weakly coupled super Yang-Mills and nothing special happens. On the other hand, if the hypermultiplet mass is tuned so that $\lambda_{\text {eff }}<0$, then $\lambda_{\text {eff }} \rightarrow 0_{-}$as $r \rightarrow \infty$. From the matrix model equations of motion in (2.5), we saw previously that the eigenvalue distribution splits into two peaks separated by approximately $2 b=-\frac{8 \pi^{2}}{\lambda_{\text {eff }}}$. In the approximation used in (2.8) the peaks have zero width. However, taking into account the subleading term in the kernel results in a nonzero width for each peak. We will show this below and in section 4 . 


\section{$3.1 \quad N$ even}

To proceed let us assume that the eigenvalue distribution is symmetric about the origin and to avoid an eigenvalue at $\sigma=0$ we choose $N$ even. Later we will consider $N$ odd. We can then divide the eigenvalues into two groups with

$$
\begin{array}{rlrl}
\sigma_{i} & =\sigma_{0}+\delta \sigma_{i} & 1 \leq i \leq N / 2 \\
\sigma_{i+N / 2} & =-\sigma_{0}+\delta \tilde{\sigma}_{i} &
\end{array}
$$

where we assume that

$$
\sum_{i}^{N / 2} \delta \sigma_{i}=\sum_{i}^{N / 2} \delta \tilde{\sigma}_{i}=0 .
$$

Letting $\lambda_{\text {eff }}^{-1} \ll-1$ the equations for $1 \leq i \leq N / 2$ become

$$
\begin{aligned}
& \frac{8 \pi^{3} N}{\lambda_{\text {eff }}}\left(\sigma_{0}+\delta \sigma_{i}\right)=\pi \sum_{j \neq i}^{N / 2}\left(2-\left(\delta \sigma_{i}-\delta \sigma_{j}\right)^{2}\right) \operatorname{coth}\left(\pi\left(\delta \sigma_{i}-\delta \sigma_{j}\right)\right) \\
& \quad+\pi N-\frac{\pi N}{2}\left(4 \sigma_{0}^{2}+4 \sigma_{0} \delta \sigma_{i}+\left(\delta \sigma_{i}\right)^{2}\right)-\pi \sum_{j=1}^{N / 2}\left(\delta \tilde{\sigma}_{j}\right)^{2}+\mathrm{O}\left(e^{-2 \sigma_{0}}\right) .
\end{aligned}
$$

If we sum (3.4) over $i$, then using the antisymmetry of the kernel we find

$$
\frac{8 \pi^{3} N}{\lambda_{\text {eff }}} \sigma_{0}=\pi N-2 \pi N \sigma_{0}^{2}-\pi \sum_{j=1}^{N / 2}\left(\delta \sigma_{j}\right)^{2}-\pi \sum_{j=1}^{N / 2}\left(\delta \tilde{\sigma}_{j}\right)^{2}+\mathrm{O}\left(e^{-2 \sigma_{0}}\right) .
$$

Substituting this expression back into (3.4) and dropping the exponentially suppressed terms we arrive at the equation

$$
\frac{\pi N}{2}\left(\delta \sigma_{i}^{2}-\frac{\lambda_{\text {eff }}}{2 \pi^{2}} \delta_{0} \delta \sigma_{i}-\overline{\delta \sigma^{2}}\right)=\pi \sum_{j \neq i}^{N / 2}\left(2-\left(\delta \sigma_{i}-\delta \sigma_{j}\right)^{2}\right) \operatorname{coth}\left(\pi\left(\delta \sigma_{i}-\delta \sigma_{j}\right)\right),
$$

where we have defined

$$
\overline{\delta \sigma^{2}} \equiv \frac{2}{N} \sum_{i=1}^{N / 2} \delta \sigma_{i}^{2}, \quad \overline{\delta \tilde{\sigma}^{2}} \equiv \frac{2}{N} \sum_{i=1}^{N / 2} \delta \tilde{\sigma}_{i}^{2}
$$

and

$$
\delta_{0} \equiv-\frac{8 \pi^{2}}{\lambda_{\text {eff }}}\left(\sigma_{0}+\frac{4 \pi^{2}}{\lambda_{\text {eff }}}\right)
$$

which from (3.5) leads to

$$
\delta_{0} \approx 1-\frac{1}{2} \overline{\delta \sigma^{2}}-\frac{1}{2} \overline{\delta \tilde{\sigma}^{2}} .
$$

In the limit $\lambda_{\text {eff }} \rightarrow 0_{-}$we can drop the linear term in $\delta \sigma_{i}$. However, later in section 4 and appendix A we will show that $\delta_{0}$ is suppressed by an inverse power of $N$ so this term can be dropped even for finite $\lambda_{\text {eff }}$ in the large $N$ limit. 
Hence, (3.6) reduces to

$$
\frac{\pi N}{2}\left(\delta \sigma_{i}^{2}+\chi\right)=\pi \sum_{j \neq i}^{N / 2}\left(2-\left(\delta \sigma_{i}-\delta \sigma_{j}\right)^{2}\right) \operatorname{coth}\left(\pi\left(\delta \sigma_{i}-\delta \sigma_{j}\right)\right),
$$

where $\chi=-\overline{\delta \sigma^{2}}$. The lefthand side of this equation can come from a free energy with the form

$$
F=\pi \frac{N}{2} \sum_{i=1}^{N / 2}\left(\frac{1}{3} \operatorname{Tr}\left(\delta \sigma_{i}\right)^{3}+\chi \operatorname{Tr}\left(\delta \sigma_{i}\right)\right)
$$

where $\delta \sigma_{i}$ are the eigenvalues for an adjoint scalar in the vector multiplet of an $\mathrm{SU}(N / 2)$ gauge theory. The first term is the contribution of a 5-dimensional Chern-Simons term at level $k=N / 2$, while the second is a Lagrange multiplier term which enforces the tracelessness condition. A similar equation can be derived for $\delta \tilde{\sigma}_{i}$ except the lefthand side of (3.10) has the opposite sign. Hence this would correspond to an $\mathrm{SU}(N / 2)$ gauge theory with a Chern-Simons term at level $k=-N / 2$.

This analysis shows that negative coupling forces the eigenvalues into two groups, essentially moving the theory far out on the Coulomb branch, such that the gauge group breaks $\mathrm{SU}(N) \rightarrow \mathrm{SU}(N / 2)_{+N / 2} \times \mathrm{SU}(N / 2)_{-N / 2} \times \mathrm{U}(1)$. The scalar field in the $\mathrm{U}(1)$ vector multiplet is $\phi=2 \sigma_{0} / R$ and we can see from (3.4) that there is a corresponding prepotential

$$
\mathcal{F}=\frac{1}{2 g_{\text {eff }}^{2}} \phi^{2}+\frac{\pi N}{24 \pi^{3}}|\phi|^{3}
$$

At the minimum where $\mathcal{F}^{\prime}=0$, we have that $\phi=-\frac{8 \pi^{2}}{g_{\text {eff }}^{2} N}$ and the $\mathrm{U}(1)$ coupling is given by

$$
\frac{1}{g^{2}}=\mathcal{F}^{\prime \prime}=-\frac{1}{g_{\mathrm{eff}}^{2}} .
$$

Hence the effective $\mathrm{U}(1)$ coupling is actually weakly positive in this regime.

As we take $r \rightarrow \infty$ the effective $\mathrm{U}(1)$ coupling is $g^{2} / r$ and flows to zero. The W-bosons charged under the $\mathrm{U}(1)$ have their masses driven to $\infty$. However, we also expect the instantons to be massless and charged under the $\mathrm{U}(1)$. Hence, the $\mathrm{U}(1)$ is lifted to $\mathrm{SU}(2)$ and the theory flows to an effective theory in the IR with $\mathrm{SU}(N / 2)_{+N / 2} \times \mathrm{SU}(N / 2)_{-N / 2}$ along with an $\mathrm{SU}(2)$ vector multiplet. This corresponds to the $(p, q)$ web shown in figure 2(b). We will comment further on the enhancement to $\mathrm{SU}(2)$ in section 5 where we discuss the contribution of instantons.

Note added. Similar results were recently obtained in the studies of phase diagrams for $5 d$ supersymmetric gauge theories using brane web constructions [22]. In particular, in one of the corners of the phase diagram for the rank $N E_{1}$ theory the authors observed a transition from the $\mathrm{SU}(N+6)_{6}$ theory to the $\mathrm{SU}\left(\frac{N+7}{2}\right)_{\frac{N+7}{2}} \times \mathrm{SU}\left(\frac{N-5}{2}\right)_{-\frac{N-5}{2}} \times \mathrm{SU}(2)$ theory for the case of odd $N$. This observation is a direct generalization of our result to the case of non-zero CS level. Each of the two $\mathrm{SU}(M)_{M}$ theories possess an $\mathrm{SU}(2)$ global symmetry at the UV fixed point. The diagonal part of these two SU(2) symmetries is then gauged and results in one $\mathrm{SU}(2)$ gauge theory and one global $\mathrm{SU}(2)$ symmetry. In our case the interpretation of the $\mathrm{SU}(2)$ factor is the same. 


\section{$3.2 \quad N$ odd}

Let us now consider the case where $N$ is odd. When the coupling is positive the distribution of the eigenvalues is symmetric about the origin, with one eigenvalue at zero, namely $\sigma_{\frac{N+1}{2}}=0$. As the coupling crosses over to the negative side, the eigenvalue at zero stays there, while the others separate into two groups of $\frac{N-1}{2}$ on either side of the origin. We let

$$
\begin{array}{rlr}
\sigma_{i} & =\sigma_{0}+\delta \sigma_{i} & 1 \leq i \leq(N-1) / 2 \\
\sigma_{i+N+1 / 2} & =\tilde{\sigma}_{0}+\delta \tilde{\sigma}_{i} &
\end{array}
$$

while assuming

$$
\sum_{i=1}^{\frac{N-1}{2}} \delta \sigma_{i}=\sum_{i=1}^{\frac{N-1}{2}} \delta \tilde{\sigma}_{i}=0
$$

Since we are considering an $\mathrm{SU}(N)$ gauge group we should impose $\tilde{\sigma}_{0}=-\sigma_{0}$. Equations (3.4) and (3.5) are then modified to

$$
\begin{aligned}
& \frac{8 \pi^{3} N}{\lambda_{\text {eff }}}\left(\sigma_{0}+\delta \sigma_{i}\right)=\pi \sum_{j \neq i}^{\frac{N-1}{2}}\left(2-\left(\delta \sigma_{i}-\delta \sigma_{j}\right)^{2}\right) \operatorname{coth}\left(\pi\left(\delta \sigma_{i}-\delta \sigma_{j}\right)\right) \\
&+\pi(N-1)-\frac{\pi(N-1)}{2}\left(4 \sigma_{0}^{2}+4 \sigma_{0} \delta \sigma_{i}+\left(\delta \sigma_{i}\right)^{2}\right)-\pi \sum_{j=1}^{\frac{N-1}{2}}\left(\delta \tilde{\sigma}_{j}\right)^{2} \\
&+2 \pi-\pi\left(\sigma_{0}^{2}+2 \sigma_{0} \delta \sigma_{i}+\left(\delta \sigma_{i}\right)^{2}\right)+\mathrm{O}\left(e^{-2 \sigma_{0}}\right), \\
& \frac{8 \pi^{3} N}{\lambda_{\text {eff }}} \sigma_{0}=\pi(N+1)-\pi(2 N-1) \sigma_{0}^{2}-\pi \frac{N+1}{2} \overline{\delta \sigma^{2}}-\pi \frac{N-1}{2} \overline{\delta \tilde{\sigma}^{2}}+\mathrm{O}\left(e^{-2 \sigma_{0}}\right),
\end{aligned}
$$

where $\overline{\delta \sigma^{2}}$ and $\overline{\delta \tilde{\sigma}^{2}}$ are defined analogously to (3.7). Solving for $\sigma_{0}$ after dropping the exponentially suppressed terms we find

$$
\sigma_{0}=-\frac{4 \pi^{2}}{\lambda_{\text {eff }}}+\frac{f\left(\lambda_{\text {eff }}\right)}{N}
$$

where

$$
f\left(\lambda_{\text {eff }}\right)=\frac{2 N}{2 N-1}\left[\frac{2 \pi^{2}(N-1)}{\lambda_{\text {eff }}}+N \sqrt{\left(\frac{2 \pi^{2}}{\lambda_{\text {eff }}}\right)^{2}+\frac{1}{4}\left(2-\overline{\delta \sigma^{2}}-\overline{\delta \tilde{\sigma}^{2}}+\frac{2}{N}\right)}\right] .
$$

Then the analog of (3.6), up to exponentially suppressed terms, is

$$
\frac{\pi(N+1)}{2}\left(\delta \sigma_{i}^{2}-\overline{\delta \sigma^{2}}\right)+2 \pi f\left(\lambda_{\text {eff }}\right) \delta \sigma_{i}=\pi \sum_{j \neq i}^{\frac{N-1}{2}}\left(2-\left(\delta \sigma_{i}-\delta \sigma_{j}\right)^{2}\right) \operatorname{coth}\left(\pi\left(\delta \sigma_{i}-\delta \sigma_{j}\right)\right) .
$$

These are the equations of motion for an $\mathrm{SU}\left(\frac{N-1}{2}\right)$ gauge theory at level $k=\frac{N+1}{2}$. Unlike the case with even $N$ we have also generated a Yang-Mills term with coupling $g^{2}=4 \pi^{2} / f\left(\lambda_{\text {eff }}\right)$. A similar analysis for the $\delta \tilde{\sigma}_{i}$ equations leads to an $\mathrm{SU}\left(\frac{N-1}{2}\right)$ gauge 
theory at level $k=-\frac{N-1}{2}$ and with the same Yang-Mills coupling. There are also two $\mathrm{U}(1)$ gauge theories with heavy $W$ particles. Hence, this corresponds to the $(p, q)$ web in figure $5(\mathrm{~b})$.

We should check the stability of this solution. To this end, consider moving $\sigma_{\frac{N+1}{2}}$ away from the origin such that we continue to satisfy the $\mathrm{SU}(N)$ condition $\frac{N-1}{2}\left(\sigma_{0}+\tilde{\sigma}_{0}\right)+\sigma_{\frac{N+1}{2}}=$ 0 . Hence, the equation of motion for $\sigma_{\frac{N+1}{2}}$ is

$$
\begin{aligned}
\frac{8 \pi^{3} N}{\lambda_{\text {eff }}} \sigma_{\frac{N+1}{2}} & =-\pi \frac{N-1}{2}\left(\sigma_{0}-\tilde{\sigma}_{0}\right)\left(2 \sigma_{\frac{N+1}{2}}-\left(\sigma_{0}+\tilde{\sigma}_{0}\right)\right)+\mathrm{O}\left(e^{-2 \sigma_{0}}\right) \\
& =-\pi N\left(\sigma_{0}-\tilde{\sigma}_{0}\right) \sigma_{\frac{N+1}{2}}+\mathrm{O}\left(e^{-2 \sigma_{0}}\right)
\end{aligned}
$$

where we have assumed that $\overline{\delta \sigma^{2}}=\overline{\delta \tilde{\sigma}^{2}}$. After dropping the exponentially suppressed terms and setting $\sigma_{0}-\tilde{\sigma}_{0}=2 \sigma_{0}$ to lowest order in $\frac{\sigma_{\frac{N+1}{2}}}{2}$, we get an equation that comes from the potential

$$
V\left(\sigma_{\frac{N+1}{2}}\right)=\pi N\left(\frac{4 \pi^{2}}{\lambda_{\text {eff }}}+\sigma_{0}\right) \sigma_{\frac{N+1}{2}}^{2}=\pi f\left(\lambda_{\text {eff }}\right) \sigma_{\frac{N+1}{2}}^{2} .
$$

Inspecting (3.19) we see that $f\left(\lambda_{\text {eff }}\right)>0$ and the solution is stable, as long as $\overline{\delta \sigma^{2}} \leq 1$.

To show that this condition is true, let us return to the equations for $\delta \sigma_{i}$ in (3.20) as $\lambda_{\text {eff }} \rightarrow 0_{-}$. In this limit we can approximate $f\left(\lambda_{\text {eff }}\right)$ by

$$
f\left(\lambda_{\text {eff }}\right) \approx-\frac{2 \pi^{2}}{\lambda_{\text {eff }}}-\frac{1-\overline{\delta \sigma^{2}}}{4} \frac{N \lambda_{\text {eff }}}{2 \pi^{2}}
$$

We can then define a new 't Hooft coupling $\lambda_{d}$ for the $\mathrm{SU}\left(\frac{N-1}{2}\right)$ gauge group, such that

$$
\lambda_{d} \equiv g^{2} \frac{N-1}{2} \approx-N \lambda_{\text {eff }}+\mathrm{O}\left(N^{-1}\right) .
$$

As $\lambda_{d} \rightarrow 0_{+}$the Yang-Mills term dominates over the Chern-Simons term and the $\delta \sigma_{i}$ approach the profile of a Gaussian matrix model, which has a width squared $\overline{\delta \sigma^{2}}=\frac{\lambda_{d}}{8 \pi^{3}}$, which satisfies the above stability condition.

Note that in the $\lambda_{d} \rightarrow 0_{+}$limit the inverse Yang-Mills coupling $f\left(\lambda_{\text {eff }}\right)$ for the $\mathrm{SU}\left(\frac{N-1}{2}\right)$ gauge theories equals half of $\sigma_{0}$. Consulting figure 5(b), the lengths of the shorter sides of the right triangles equal $\sigma_{0}$. Hence, the inverse coupling is half the length of the D5 branes.

\subsection{The Chern-Simons levels directly from field theory}

In this subsection we derive the Chern-Simons levels directly in a field theory calculation. In particular, the shifts of the Chern-Simons levels described above come from the decoupling of massive fermions. For definiteness we consider the case of even $N$ in this section. All derivations presented below can be easily modified for the case of odd $N$.

Let us write down the part of the Lagrangian quadratic in the fermions for a vector multiplet in a $5 d$ Euclidian theory [23, 24]:

$$
\mathcal{L}_{\mathrm{vec}} \sim \frac{1}{g_{\mathrm{YM}}^{2}} \operatorname{Tr}\left(i \lambda^{\dagger} \Gamma^{\mu} D_{\mu} \lambda-\lambda^{\dagger}[\sigma, \lambda]\right),
$$


which gives the equation of motion

$$
i \Gamma^{\mu} D_{\mu} \lambda-[\sigma, \lambda]=0 .
$$

We next move onto the Coulomb branch by giving the scalar $\sigma$ the expectation value

$$
\langle\sigma\rangle=\left[\begin{array}{cc}
\sigma_{0} \mathbb{I}_{N / 2 \times N / 2} & 0 \\
0 & -\sigma_{0} \mathbb{I}_{N / 2 \times N / 2}
\end{array}\right],
$$

and also write the adjoint fermion $\lambda$ in block form as

$$
\lambda=\left[\begin{array}{ll}
\lambda^{11} & \lambda^{12} \\
\lambda^{21} & \lambda^{22}
\end{array}\right] .
$$

The blocks $\lambda^{11}$ and $\lambda^{22}$ correspond to the adjoint fermions of the $\mathrm{SU}(N / 2)$ subgroups while $\lambda^{12}$ and $\lambda^{21}$ are in the bifundamental representations $(N / 2, \bar{N} / 2)$ and $(\bar{N} / 2, N / 2)$ respectively. Equation (3.26) then splits into the Dirac equations

$$
\begin{aligned}
i \Gamma^{\mu} D_{\mu} \lambda^{11} & =0, & i \Gamma^{\mu} D_{\mu} \lambda^{22} & =0, \\
\left(i \Gamma^{\mu} D_{\mu}-2 \sigma_{0}\right) \lambda^{12} & =0, & \left(i \Gamma^{\mu} D_{\mu}+2 \sigma_{0}\right) \lambda^{21} & =0 .
\end{aligned}
$$

Hence, the $\lambda^{12}$ fermions acquire a negative mass $-2 \sigma_{0}$, the $\lambda^{21}$ fermions get a positive mass $+2 \sigma_{0}$, while $\lambda^{11}$ and $\lambda^{22}$ stay massless. ${ }^{3}$ As was shown in [25], integrating out the massive fermions leads to a shift of the Chern-Simons level

$$
\delta k=-\operatorname{sign}(m) \frac{C_{3}(R)}{2} .
$$

Applying this to our case and using that $C_{3}(R)$ is $+1(-1)$ for a fundamental (antifundamental) representation, we get the following level shifts from the decoupled fermions:

$$
\begin{aligned}
& \lambda^{12}: \quad \delta k_{1}^{12}=\frac{1}{2} \frac{N}{2}, \quad \delta k_{2}^{12}=\frac{1}{2}\left(-\frac{N}{2}\right), \\
& \lambda^{21}: \quad \delta k_{1}^{21}=-\frac{1}{2}\left(-\frac{N}{2}\right), \quad \delta k_{2}^{21}=-\frac{1}{2} \frac{N}{2},
\end{aligned}
$$

which combine to give

$$
\delta k_{1}=\frac{N}{2}, \quad \delta k_{2}=-\frac{N}{2},
$$

consistent with the result in section 3.1 .

Note that if the bifundamental fermions had come from a hypermultiplet the shift in the levels would have been the opposite. This is clear from the fermion quadratic term for an adjoint hypermultiplet,

$$
\mathcal{L}_{\text {hyp }} \sim-2 i \psi^{\dagger} \Gamma^{\mu} D_{\mu} \psi-2 \psi^{\dagger}[\sigma, \psi],
$$

where one can see that the relative sign between the kinetic term and the Yukawa term is the opposite of (3.26).

\footnotetext{
${ }^{3}$ Since we work with a Euclidian metric the sign in front of the mass term is opposite to the one used in $[25]$.
} 


\section{$4 \mathrm{SU}(N / 2)_{N / 2}$ Chern-Simons}

In this section we consider the $\mathrm{SU}(N / 2)_{N / 2}$ gauge theory with no Yang-Mills term, whose $(p, q)$ web is shown in figure 3 and its eigenvalue equations are given in (3.10). We also consider what happens as one turns on the Yang-Mills term.

The Chern-Simons level $k$ has to satisfy $-N / 2 \leq k \leq N / 2$ in order to have a nontrivial fixed point, hence the theory we consider is the maximum in this range. In the large $N$ limit, sitting at the maximum level pushes the support for the eigenvalue density out to $-\infty$. Hence the eigenvalue equations (3.10) reduce to an integral equation with the form

$$
\sigma^{2}+\chi=f_{-\infty}^{b} d \sigma^{\prime} \rho\left(\sigma^{\prime}\right)\left(2-\left(\sigma-\sigma^{\prime}\right)^{2}\right) \operatorname{coth}\left(\pi\left(\sigma-\sigma^{\prime}\right)\right)
$$

where the density is normalized to $\int_{-\infty}^{b} d \sigma \rho(\sigma)=1$ and the integration endpoint $b$ is positive of order 1 . Since the integration region extends all the way to $-\infty$, and if we assume that the eigenvalue density falls off exponentially as $\sigma^{\prime} \rightarrow-\infty$, then in the limit $\sigma \rightarrow-\infty,(4.1)$ simplifies to

$$
\sigma^{2}+\chi=\int_{-\infty}^{b} d \sigma^{\prime} \rho\left(\sigma^{\prime}\right)\left(-2+\left(\sigma-\sigma^{\prime}\right)^{2}\right)=\sigma^{2}-2+\int_{-\infty}^{b} d \sigma^{\prime} \rho\left(\sigma^{\prime}\right)\left(\sigma^{\prime}\right)^{2}
$$

where we used (3.3). Hence, the Lagrange multiplier is

$$
\chi=-2+\int_{-\infty}^{b} d \sigma \rho(\sigma) \sigma^{2} .
$$

If we integrate both sides of (4.1) we also have

$$
\int_{-\infty}^{b} d \sigma \rho(\sigma) \sigma^{2}+\chi=\int_{-\infty}^{b} d \sigma \rho(\sigma) f_{-\infty}^{b} d \sigma^{\prime} \rho\left(\sigma^{\prime}\right)\left(2-\left(\sigma-\sigma^{\prime}\right)^{2}\right) \operatorname{coth}\left(\pi\left(\sigma-\sigma^{\prime}\right)\right)=0,
$$

since the integrand is antisymmetric under the exchange of $\sigma$ and $\sigma^{\prime}$. Combining this with (4.3) we find

$$
\int_{-\infty}^{b} d \sigma \rho(\sigma) \sigma^{2}=1, \quad \chi=-1 .
$$

The first result tells us that the width of the distribution is 1 in the large- $N$ limit. Since the distribution of the two sets of eigenvalues is symmetric, meaning that

$$
\frac{2}{N} \sum_{j=1}^{N / 2}\left(\delta \sigma_{j}\right)^{2}=\frac{2}{N} \sum_{j=1}^{N / 2}\left(\delta \tilde{\sigma}_{j}\right)^{2} \approx \int_{-\infty}^{b} d \sigma \rho(\sigma) \sigma^{2}=1,
$$

it follows from (3.5), (3.8), and (4.5) that it is consistent to set $\delta_{0}=0$ in (3.6). In appendix A we show that for finite $N$ the width squared for the solution to (3.10) is precisely

$$
\frac{2}{N} \sum_{j=1}^{N / 2}\left(\delta \sigma_{j}\right)^{2}=1-\frac{2}{N}
$$

hence from (3.5) we see that $\delta_{0} \approx 1 / N$ and can be ignored in the large- $N$ limit. 


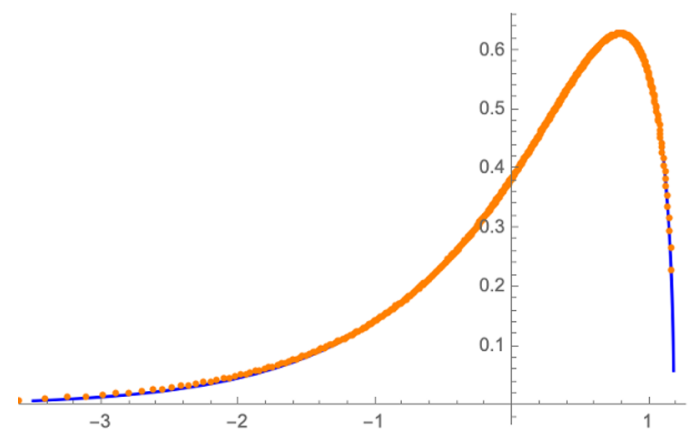

Figure 6. Eigenvalue density for $\mathrm{SU}(N / 2)_{N / 2}$ Chern-Simons theory with $N / 2=500$. The orange dots are the numerical result while the blue curve is a fit to the function $a \sqrt{b-\sigma} e^{c \sigma}$ with a best fit at $a=0.3566, b=1.182$ and $c=1.308$.

While we expect $\rho(\sigma)$ to have a square root branch cut and an exponential fall off, it does not seem possible to solve for $\rho(\sigma)$ analytically in (3.10). However, we can find the solution numerically. Figure 6 shows the distribution of eigenvalues with $N / 2=500$. We also show a best fit for the simplest function meeting the stated criteria, $f(\sigma)=a \sqrt{b-\sigma} e^{c \sigma}$. The best fit parameters are given in the figure.

The exponential fall off on the tail of the distribution can be justified as follows. On the tail the eigenvalues are generically widely separated from each other, in which case we can make the approximation $\cosh \left(\pi\left(\sigma-\sigma^{\prime}\right)\right) \approx \operatorname{sign}\left(\sigma-\sigma^{\prime}\right)$. If we make this replacement in (4.1) then the integral equation takes the form

$$
\sigma^{2}+\chi=f_{-\infty}^{b} d \sigma^{\prime} \rho\left(\sigma^{\prime}\right)\left(2-\left(\sigma-\sigma^{\prime}\right)^{2}\right) \operatorname{sign}\left(\sigma-\sigma^{\prime}\right)
$$

Taking three derivatives on both sides of the equation then gives

$$
0=4 \rho(\sigma)-4 \rho^{\prime \prime}(\sigma), \quad \sigma<b
$$

The only allowable solution is $\rho(\sigma)=A e^{\sigma-b}$. The coefficient $A$ and the endpoint $b$ are determined by normalizing the density and setting $\int_{-\infty}^{b} d \sigma \rho(\sigma) \sigma=0$, which gives $A=$ $b=1$. Figure 7 shows the numerical results on the tail compared with this exponential approximation, clearly showing a good fit as one moves out along the tail.

It is interesting to turn on the Yang-Mills coupling in this theory. The addition of the Yang-Mills action adds the term $\frac{16 \pi^{2} r}{g_{Y M}^{N}} \sigma$ to the left hand side of (4.1). It also leads to a finite integration region on the right hand side of this equation. Unlike the cases when $-N / 2<k<N / 2$, it is not possible to pass through to the negative coupling side when $k= \pm N / 2$. This can be seen from the $(p, q)$ web, but also from (4.2), since the right hand side cannot generate a linear term in $\sigma$.

We can then find the dependence of the free energy on the Yang-Mills coupling, assuming that it is large. At infinite coupling the free energy is $F=C_{0} N^{2}$ where $C_{0}$ is a constant of order 1 . If we then modify the coupling such that $\frac{4 \pi^{3} r}{g_{\mathrm{YM}}^{2}} \ll 1$, then the new 


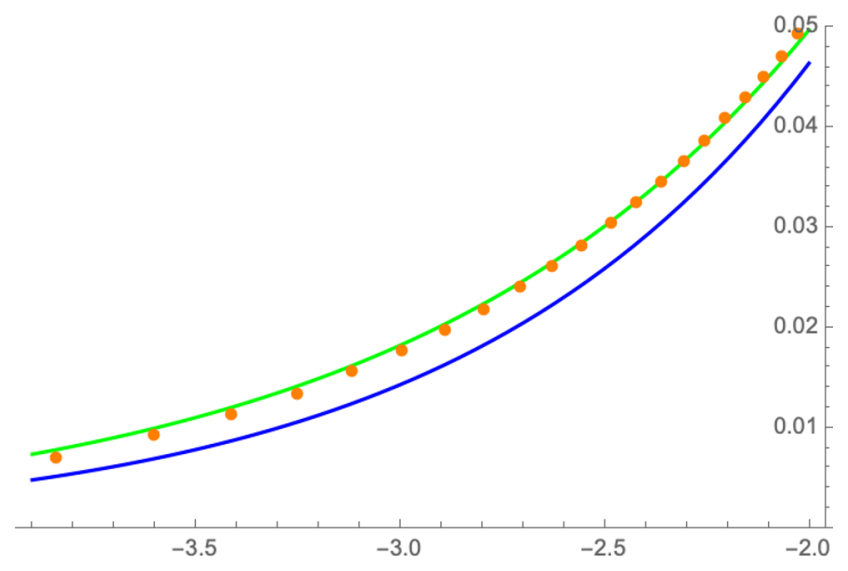

Figure 7. Eigenvalue density for $\mathrm{SU}(N / 2)_{N / 2}, N / 2=500$, on the tail. The orange dots are the numerical result while the green curve is the approximate density $\rho(\sigma)=e^{\sigma-1}$. The blue curve is the fit shown in figure 6.

free energy is approximately

$$
\begin{aligned}
F & \approx C_{0} N^{2}+\left\langle\frac{4 \pi^{3} r}{g_{\mathrm{YM}}^{2}} \sum_{i=1}^{N / 2} \sigma_{i}^{2}\right\rangle \\
& \approx C_{0} N^{2}+\frac{4 \pi^{3} r}{g_{\mathrm{YM}}^{2}} \frac{N}{2} \int_{-\infty}^{b} d \sigma \rho(\sigma) \sigma^{2}=C_{0} N^{2}+\frac{2 \pi^{3} r N}{g_{\mathrm{YM}}^{2}}
\end{aligned}
$$

where we used (4.5) in the last step.

\section{Instantons at large $N$ in $5 \mathrm{D}$}

In this section we consider the effect of instantons on the large $N$ approximation. While such a study has been carried out in four dimensions, the same has previously not been done in five. Here we will show that the instantons are exponentially suppressed in the large $N$ limit and so can be safely ignored.

As in the case of four dimensions with eight supersymmetries, we need to worry about the contributions of instantons if the effective coupling is negative. Normally, instantons are suppressed by a factor of $e^{-1 / g^{2}}$, which in the large $N$ limit with fixed 't Hooft constant $\lambda$ can be ignored. However, if the effective coupling is negative, then the instantons could be enhanced at large $N$. Such a scenario was considered in four dimensions, where it was shown by a careful analysis that instantons are still suppressed in the large $N$ limit [6].

In five dimensions the instantons are actually particles that traverse world lines. On $S^{5}$ one expects the instantons to be localized on the Reeb orbits fibered over the $C P^{2}$ fixed points at $\left(z_{1}, z_{2}, z_{3}\right)=(1,0,0),(0,1,0)$ and $(0,0,1)$. Their contribution to the partition function is conjectured to be [8]

$$
Z_{\mathbb{R}^{4} \times S^{1}}^{\text {inst }}\left(i \sigma, i \mu, \frac{2 \pi}{\omega_{2}}, \omega_{1}+\omega_{2}, \omega_{3}\right) Z_{\mathbb{R}^{4} \times S^{1}}^{\text {inst }}\left(i \sigma, i \mu, \frac{2 \pi}{\omega_{1}}, \omega_{3}+\omega_{1}, \omega_{2}\right) Z_{\mathbb{R}^{4} \times S^{1}}^{\text {inst }}\left(i \sigma, i \mu, \frac{2 \pi}{\omega_{3}}, \omega_{1}+\omega_{3}, \omega_{2}\right)
$$


where $Z_{\mathbb{R}^{4} \times S^{1}}^{\text {inst }}\left(i \sigma, i \mu, \beta, \epsilon_{1}, \epsilon_{2}\right)$ is the Nekrasov partition function for the $\mathcal{N}=1^{*}$ theory on $\mathbb{R}^{4} \times S^{1}$. The $\omega_{i}$ are three squashing parameters on the sphere which we keep in order to regulate the answer. On the round sphere $\omega_{i}=1$. Here we only consider the one instanton partition function, whose contribution from the first fixed point is given by

$$
\begin{aligned}
Z_{\mathbb{R}^{4} \times S^{1}}^{1}\left(i \sigma, i \mu, \frac{2 \pi}{\omega_{2}}, \omega_{1}+\omega_{2}, \omega_{3}\right) \\
=\frac{e^{-\frac{8 \pi^{3} r}{g_{\mathrm{YM}}^{2} \omega_{2}}} \sinh \left(\frac{\pi}{\omega_{2}}\left(\sigma_{j i}+\hat{\mu}+i \omega_{3}\right)\right) \sinh \left(\frac{\pi}{\omega_{2}}\left(\sigma_{i j}+\hat{\mu}+i\left(\omega_{1}+\omega_{2}\right)\right)\right)}{\sin \left(\frac{\pi \omega_{1}}{\omega_{2}}\right) \sin \left(\frac{\pi \omega_{3}}{\omega_{2}}\right)} \\
\quad \times \sum_{i}^{N} \prod_{j \neq i}^{N} \frac{\sinh \left(\frac{\pi}{\omega_{2}}\left(\sigma_{j i}+\hat{\mu}+i \Delta\right)\right) \sinh \left(\frac{\pi}{\omega_{2}}\left(\sigma_{i j}+\hat{\mu}\right)\right)}{\sinh \left(\frac{\pi}{\omega_{2}}\left(\sigma_{j i}+i \Delta\right)\right) \sinh \left(\frac{\pi}{\omega_{2}} \sigma_{i j}\right)}
\end{aligned}
$$

where $\hat{\mu}=\mu-\frac{i}{2}\left(\omega_{1}+\omega_{2}+\omega_{3}\right)[26]$ and $\Delta=\omega_{1}+\omega_{2}+\omega_{3}$. If we assume that $\mu \gg\left|\sigma_{i j}\right|$ then we can approximate (5.2) as

$$
\begin{aligned}
& Z_{\mathbb{R}^{4} \times S^{1}}^{1}\left(i \sigma, i \mu, \frac{2 \pi}{\omega_{2}}, \omega_{1}+\omega_{2}, \omega_{3}\right) \\
& \approx \frac{e^{-\frac{8 \pi^{3} r}{g_{\mathrm{YM}}^{2} \omega_{2}}+\frac{2 \pi N \mu}{\omega_{2}}}}{2^{2 N} \sin \left(\frac{\pi \omega_{1}}{\omega_{2}}\right) \sin \left(\frac{\pi \omega_{3}}{\omega_{2}}\right)} \sum_{i}^{N} \prod_{j \neq i}^{N} \frac{1}{\sinh \left(\frac{\pi}{\omega_{2}}\left(\sigma_{j i}+i \Delta\right)\right) \sinh \left(\frac{\pi}{\omega_{2}} \sigma_{i j}\right)} \\
& =\frac{e^{-\frac{8 \pi^{3} N}{\lambda_{\text {eff }} \omega_{2}}}}{2^{2 N} \sin \left(\frac{\pi \omega_{1}}{\omega_{2}}\right) \sin \left(\frac{\pi \omega_{3}}{\omega_{2}}\right)} \sum_{i}^{N} \prod_{j \neq i}^{N} \frac{1}{\sinh \left(\frac{\pi}{\omega_{2}}\left(\sigma_{j i}+i \Delta\right)\right) \sinh \left(\frac{\pi}{\omega_{2}} \sigma_{i j}\right)} .
\end{aligned}
$$

The leading factor is divergent as we approach the round sphere. However, if we combine this contribution with those from the other two fixed points and take the limit $\omega_{i} \rightarrow 1$ we find that the divergence cancels and there is an overall contribution of order $N^{2}$. Hence, the full one instanton contribution has the form

$$
Z_{S^{5}}^{1}(i \sigma) \sim N^{2} 2^{-2 N} e^{-\frac{8 \pi^{3} N}{\lambda_{\mathrm{eff}}}} \sum_{i}^{N} \prod_{j \neq i}^{N} \frac{1}{\sinh ^{2}\left(\pi \sigma_{j i}\right)} .
$$

If $\lambda_{\text {eff }}<0$ then it would appear that the instanton contribution blows up in the large $N$ limit. However, if we assume that the eigenvalues have the distribution in (3.2) and (3.8), and further assume that $\delta \tilde{\sigma}_{i}=-\delta \sigma_{i}$ then (5.4) becomes

$$
\begin{aligned}
Z_{S^{5}}^{1}(i \sigma) & \sim N^{2} 2^{-N} e^{-\frac{8 \pi^{3} N}{\lambda_{\mathrm{eff}}}} \sum_{i}^{N / 2} \prod_{j \neq i}^{N / 2} \frac{e^{-2 \pi\left(\delta \sigma_{i}+2 \sigma_{0}\right)}}{\sinh ^{2}\left(\pi \delta \sigma_{j i}\right)} \\
& =N^{2} 2^{-N} \sum_{i}^{N / 2} \prod_{j \neq i}^{N / 2} \frac{e^{-2 \pi \delta \sigma_{i}}}{\sinh ^{2}\left(\pi \delta \sigma_{j i}\right)} .
\end{aligned}
$$

While we are unaware of a way to find the product in (5.5) analytically, we can do it numerically in the limit where $\lambda_{\text {eff }} \rightarrow 0_{-}$. If we choose the index $i$ such that the product 
is a maximum, we find that

$$
2^{-N} \prod_{j \neq i}^{N / 2} \frac{e^{-2 \pi \delta \sigma_{i}}}{\sinh ^{2}\left(\pi \delta \sigma_{j i}\right)} \sim N^{-2} \exp (-2.03 N) .
$$

Hence, there is an exponential suppression in $N$ and the instantons can be ignored. A further explanation of the exponential behavior as well as the prefactor is given in appendix B.

Note that in the second line of (5.5) the $\lambda_{\text {eff }}$ dependence has canceled out. This is a manifestation of the fact that the instanton particles are massless when $\lambda_{\text {eff }}<0$ and the $\mathrm{U}(1)$ gauge group is enhanced to $\mathrm{SU}(2)$. The instanton suppression is instead due to the finite spread of the eigenvalues as seen in figure 6 . In other words, it is due to the suppression coming from the individual $\mathrm{SU}(N / 2)_{ \pm N / 2}$ gauge theories.

\section{$6 \operatorname{USp}(2 N)$ gauge theories}

In this section we investigate five-dimensional $\operatorname{USp}(2 N)$ supersymmetric gauge theories. These theories are notable because with an appropriate set of massless hypermultiplets they can have a superconformal fixed point that is dual to an $A d S_{6}$ background [10, 11]. An interesting question is what happens when passing through the fixed point as one varies the inverse Yang-Mills coupling.

We start with a gauge theory that contains the $\operatorname{USp}(2 N)$ vector multiplet, one antisymmetric hypermultiplet with mass $m_{A}$, and eight fundamental hypermultiplets with masses $m_{k}, k=1 \ldots 8$. The matrix integral for the partition function is derived using (2.1), (2.2) and (2.3). In the large $N$ limit this integral is dominated by a saddle point which satisfies the equations

$$
\begin{aligned}
\frac{16 \pi^{3} N}{\lambda} \sigma_{i}=\pi \sum_{j \neq i} & {\left[\left(2-\left(\sigma_{i} \pm \sigma_{j}\right)^{2}\right) \operatorname{coth}\left(\pi\left(\sigma_{i} \pm \sigma_{j}\right)\right)\right]+2 \pi\left(2-4 \sigma_{i}^{2}\right) \operatorname{coth}\left(2 \pi \sigma_{i}\right) } \\
+\frac{\pi}{2} \sum_{j \neq i}\left[\left(\frac{1}{4}+\left(\sigma_{i} \pm \sigma_{j}+m_{A}\right)^{2}\right) \tanh \left(\pi\left(\sigma_{i} \pm \sigma_{j}+m_{A}\right)\right)\right. & \\
& \left.\quad+\left(\frac{1}{4}+\left(\sigma_{i} \pm \sigma_{j}-m_{A}\right)^{2}\right) \tanh \left(\pi\left(\sigma_{i} \pm \sigma_{j}-m_{A}\right)\right)\right] \\
+ & \frac{\pi}{2} \sum_{k=1}^{8}\left(\left(\frac{1}{4}+\left(\sigma_{i}+m_{k}\right)^{2}\right) \tanh \left(\pi\left(\sigma_{i}+m_{k}\right)\right)+\left(\frac{1}{4}+\left(\sigma_{i}-m_{k}\right)^{2}\right) \tanh \left(\pi\left(\sigma_{i}-m_{k}\right)\right)\right) .
\end{aligned}
$$

Here $\sigma_{i}, i=1, \ldots, N$, are half of the eigenvalues of the matrix while the other half are at $-\sigma_{i}$. Without loss of generality we can assume that $\sigma_{i}>0$. Also the \pm signs mean that we sum each term with both a "+" and a "-". The 't Hooft coupling $\lambda$ is restricted to be positive.

If we set all hypermultiplet masses to zero and assume that $\lambda \gg 1$, then (6.1) reduces to

$$
\frac{16 \pi^{2} N}{\lambda} \sigma_{i}=\sum_{j \neq i}\left[\frac{9}{4} \operatorname{sign}\left(\sigma_{i}-\sigma_{j}\right)\right]+\frac{9}{4}(N-1)+6,
$$


whose solution is

$$
\sigma_{j}=\frac{3 \lambda}{32 \pi^{2} N}(3 j+1)
$$

This is very similar to the solution for an $\mathrm{SU}(N)$ gauge theory with a massless adjoint hypermultiplet at strong coupling $[19,24,27]$ and leads to a free energy that scales as $-\lambda N^{2}$.

\subsection{Negative $\lambda_{\text {eff }}$ with decoupled fundamental hypermultiplets}

Now let us decouple one or more of the fundamental hypermultiplets by taking their masses to be large. At the same time let us set all other masses to zero. In this case we find an effective coupling that takes the form

$$
\frac{1}{\lambda_{\text {eff }}}=\frac{1}{\lambda}-\frac{1}{8 \pi^{2}} \frac{1}{N} \sum_{i=N_{f}+1}^{8} m_{i}
$$

where $N_{f}$ is the number of fundamental massless hypermultiplets. Naively, decoupling a fundamental hypermultiplet has no effect on the effective coupling because of the $1 / N$ factor. However, we can let the mass of one or more of the fundamentals scale with $N$, such that we push $\lambda_{\text {eff }}$ into negative territory. We then end up with a theory with a massless antisymmetric hypermultiplet, $N_{f}<8$ massless fundamental hypermultiplets and an effective coupling which can be either positive or negative

If we let $\lambda_{\text {eff }}<0$, then the central potential repels the eigenvalues away from the origin and, as in the $\mathrm{SU}(N)$ case, we expect that the eigenvalues will group around points far away from the origin. Using the large separation limit and assuming that there are $N_{f}<8$ remaining massless fundamentals, (6.1) reduces to

$$
\frac{16 \pi^{2} N}{\lambda_{\text {eff }}} \sigma_{i}=\frac{9}{4} \sum_{j \neq i} \operatorname{sign}\left(\sigma_{i}-\sigma_{j}\right)+\frac{9}{4}(N-1)+4+\frac{1}{4} N_{f}-\left(8-N_{f}\right) \sigma_{i}^{2} .
$$

This has the solution $\sigma_{i}=\sigma_{0}$, where

$$
\sigma_{0}=\frac{8 \pi^{2} N}{\left|\lambda_{\text {eff }}\right|\left(8-N_{f}\right)}+\frac{1}{2} \sqrt{\left(\frac{16 \pi^{2} N}{\left|\lambda_{\text {eff }}\right|\left(8-N_{f}\right)}\right)^{2}+\frac{9 N+7+N_{f}}{8-N_{f}}} \approx \frac{16 \pi^{2} N}{\left|\lambda_{\text {eff }}\right|\left(8-N_{f}\right)} .
$$

Similar to the $\mathrm{SU}(N)$ case, the solution to (6.1) actually has a finite size distribution of eigenvalues around the peak at $\sigma_{0}$. If we define

$$
\sigma_{i}=\sigma_{0}+\delta \sigma_{i}, \quad \sum_{i} \delta \sigma_{i}=0
$$

then the saddle point equation (6.1) becomes

$$
\begin{gathered}
\pi\left(8-N_{f}\right) \delta \sigma_{i}^{2}+\left(\frac{16 \pi^{3} N}{\lambda_{\text {eff }}}+2 \pi \sigma_{0}\left(8-N_{f}\right)\right) \delta \sigma_{i}+\frac{16 \pi^{3} N}{\lambda_{\text {eff }}} \sigma_{0}+\pi \sigma_{0}^{2}\left(8-N_{f}\right) \\
=\pi \sum_{i \neq j}\left[\left(2-\delta \sigma_{i j}^{2}\right) \operatorname{coth}\left(\pi \delta \sigma_{i j}\right)+\left(\frac{1}{4}+\delta \sigma_{i j}^{2}\right) \tanh \left(\pi \delta \sigma_{i j}\right)\right]
\end{gathered}
$$


where $\delta \sigma_{i j} \equiv \delta \sigma_{i}-\delta \sigma_{j}$. Summing equations (6.8) we can find

$$
\sigma_{0}=\frac{16 \pi^{2} N}{\left|\lambda_{\text {eff }}\right|\left(8-N_{f}\right)}+\delta_{0} \frac{\left|\lambda_{\text {eff }}\right|}{8 \pi^{2}}, \quad \delta_{0}=\frac{N_{f}-8}{2 N} \overline{\delta \sigma^{2}} .
$$

Taking the large $N$ limit we arrive at the following singular integral equation

$$
\begin{aligned}
\frac{16 \pi^{2}}{\left|\lambda_{\text {eff }}\right|} \delta \sigma+\frac{\left(8-N_{f}\right)}{N} \delta \sigma^{2}+2 \delta_{0}=\int d\left(\delta \sigma^{\prime}\right) \rho\left(\delta \sigma^{\prime}\right) & {\left[\left(2-\left(\delta \sigma-\delta \sigma^{\prime}\right)^{2}\right) \operatorname{coth}\left(\pi\left(\delta \sigma-\delta \sigma^{\prime}\right)\right)+\right.} \\
& \left.\left(\frac{1}{4}+\left(\delta \sigma-\delta \sigma^{\prime}\right)^{2}\right) \tanh \left(\pi\left(\delta \sigma-\delta \sigma^{\prime}\right)\right)\right] .
\end{aligned}
$$

These kind of equations have already been analyzed in the context of five-dimensional YM-CS theory in [28].

If we consider weak negative coupling $\left|\lambda_{\text {eff }}\right| \ll 1$, then (6.10) describes an $\mathrm{SU}(N)$ theory with a small positive 't Hooft coupling $\left|\lambda_{\text {eff }}\right|$ and a Chern-Simons level at $\left(8-N_{f}\right)$. In this approximation the righthand side of the saddle point equation is dominated by the Yang-Mills term, creating a deep central potential for the eigenvalues. This leads to a small support for the eigenvalues, such that $\left|\delta \sigma^{\prime}-\delta \sigma\right| \ll 1$. Hence, we can approximate the saddle point equation by

$$
\frac{16 \pi^{3}}{\left|\lambda_{\mathrm{eff}}\right|} \delta \sigma+\frac{\pi\left(8-N_{f}\right)}{N} \delta \sigma^{2}+2 \pi \delta_{0}=2 \int d\left(\delta \sigma^{\prime}\right) \frac{\rho\left(\delta \sigma^{\prime}\right)}{\delta \sigma-\delta \sigma^{\prime}} .
$$

This equation is solved by the eigenvalue density

$$
\rho(\delta \sigma)=\frac{a}{\pi}(\delta \sigma+\kappa+b) \sqrt{(a b)^{-1}-(\delta \sigma+\kappa-b)^{2}},
$$

where we have introduced the parameters

$$
a=\frac{\pi\left(8-N_{f}\right)}{2 N}, \quad \kappa=\frac{8 \pi^{2} N}{\left|\lambda_{\text {eff }}\right|\left(8-N_{f}\right)}, \quad \mu=-\frac{2 N}{8-N_{f}} \delta_{0},
$$

and the constant $b$ satisfies

$$
b\left(\kappa^{2}+\mu-b^{2}\right)=\frac{1}{2 a} .
$$

Now imposing the $\mathrm{SU}(N)$ condition $\int d(\delta \sigma) \delta \sigma \rho(\delta \sigma)=0$ we obtain

$$
\mu=(\kappa-3 b)(b-\kappa), \quad b^{2}(\kappa-b)=\frac{1}{8 a} .
$$

It can be checked that with this choice of $\mu$ the relation (6.9) for $\delta_{0}$ is automatically satisfied. Instead of solving for $b$ exactly we can use that $a \ll 1$ and $\kappa \gg 1$. Then if we want the distribution (6.12) to have small support we should assume that $b \approx \kappa$, which leads to

$$
b \approx \kappa-\frac{1}{8 a \kappa^{2}}, \quad \mu \approx \frac{1}{4 a \kappa} .
$$




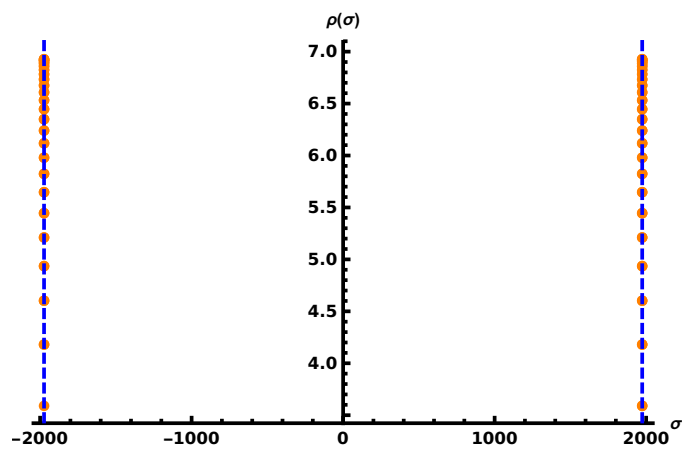

(a) Density for $\lambda_{\text {eff }}=-1$

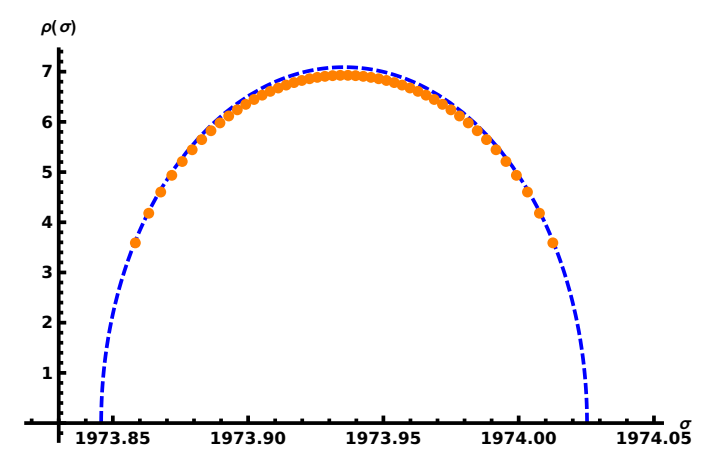

(b) Zooming in on the right peak

Figure 8. Eigenvalue density $\rho(\delta \sigma)$ for $\lambda_{\text {eff }}=-1, N=50$ and $N_{f}=4$. The dashed lines represent the analytical solutions (6.6) and (6.17).

Finally, substituting this solution back into the eigenvalue density (6.12) we find

$$
\rho(\delta \sigma) \approx \frac{a}{\pi}\left(\delta \sigma+2 \kappa-\frac{1}{8 a \kappa^{2}}\right) \sqrt{(a \kappa)^{-1}-\left(\delta \sigma+\frac{1}{8 a \kappa^{2}}\right)^{2}} \approx \frac{8 \pi^{2}}{\left|\lambda_{\text {eff }}\right|} \sqrt{\frac{\left|\lambda_{\text {eff }}\right|}{4 \pi^{3}}-\delta \sigma^{2}} .
$$

The last approximation is a semicircle distribution, as one would expect for a weak 't Hooft coupling when the right hand side of (6.11) is dominated by the Yang-Mills term and the Chern-Simons term can be ignored.

In figure 8 we show the analytic solution described above compared with the numerical solution to (6.1) at negative coupling. As we see, both the approximate solution in (6.6) and the refined solution in (6.17) work very well. It is crucial to keep the subleading terms in (6.6) in order to match (6.17) with the numerical solution, as shown in the right plot in figure 8.

\subsection{Negative $\lambda_{\text {eff }}$ with a decoupled antisymmetric hypermultiplet}

We can also give a large mass to the antisymmetric hypermultiplet. In this case the effective coupling is given by

$$
\frac{1}{\lambda_{\text {eff }}}=\frac{1}{\lambda}-\frac{1}{8 \pi^{2}}\left(2 m_{A}+\frac{1}{N} \sum_{i=N_{f}+1}^{8} m_{i}\right)
$$

and the saddle point equation for the matrix integral turns into

$$
\begin{aligned}
\frac{16 \pi^{3} N}{\lambda_{\text {eff }}} \sigma_{i}=\pi \sum_{j \neq i} & \left(2-\left(\sigma_{i} \pm \sigma_{j}\right)^{2}\right) \operatorname{coth}\left(\pi\left(\sigma_{i} \pm \sigma_{j}\right)\right)+2 \pi\left(2-4 \sigma_{i}^{2}\right) \operatorname{coth}\left(2 \pi \sigma_{i}\right) \\
& +\pi N_{f}\left(\frac{1}{4}+\sigma_{i}^{2}\right) \tanh \left(\pi \sigma_{i}\right) .
\end{aligned}
$$

These equations are very close to the ones obtained by decoupling an adjoint hypermultiplet in $\mathcal{N}=1^{*} \mathrm{SYM}$ for an $\mathrm{SU}(N)$ gauge theory. As in that case we assume $-\lambda_{\text {eff }} \ll 1$ 
which leads to two widely separated peaks in the eigenvalue distribution. Again we make the ansatz

$$
\sigma_{i}=\sigma_{0}+\delta \sigma_{i}, \quad \sum_{i=1}^{N} \delta \sigma_{i}=0
$$

in which case summing over $i$ in (6.19) leads to the equation

$$
\frac{16 \pi^{2} N}{\lambda_{\text {eff }}} \sigma_{0}=2 N+2+\frac{N_{f}}{4}-4\left(N+1-\frac{N_{f}}{4}\right) \sigma_{0}^{2}-\left(2+\frac{4-N_{f}}{N}\right) \sum_{j=1}^{N}\left(\delta \sigma_{j}\right)^{2}+\mathrm{O}\left(e^{-2 \sigma_{0}}\right),
$$

whose solution is

$$
\sigma_{0}=-\frac{4 \pi^{2}}{\lambda_{\text {eff }}}+\frac{f_{U}\left(\lambda_{\text {eff }}\right)}{N}
$$

where

$$
f_{U}\left(\lambda_{\text {eff }}\right) \approx \frac{\pi^{2}}{\lambda_{\text {eff }}}\left(4-N_{f}\right)+\frac{\lambda_{\text {eff }}}{8 \pi^{2}} N\left(1-\overline{\delta \sigma^{2}}\right)
$$

Then the saddle point equation (6.19) reduces to

$$
\begin{aligned}
\pi(N & \left.+4-N_{f}\right)\left(\delta \sigma_{i}^{2}-\overline{\delta \sigma^{2}}\right)+4 \pi\left(\frac{2 \pi^{2}}{\lambda_{\text {eff }}}\left(N_{f}-4\right)+f_{U}\left(\lambda_{\text {eff }}\right)\right) \delta \sigma_{i} \\
& =\pi \sum_{j \neq i}\left(2-\delta \sigma_{i j}^{2}\right) \operatorname{coth}\left(\pi \delta \sigma_{i j}\right)
\end{aligned}
$$

This is the equation for an $\mathrm{SU}(N)$ gauge theory with Chern-Simons level $k=N+4-N_{f}$. If $N_{f}<4$ then the Chern-Simons level is greater than $N$ with a positive Yang-Mills term which is more in line with the $\mathrm{SU}(2 N+1)$ case. In figure 9 (a) we show numerical solutions both for equations (6.19) (orange dots) and (6.24) (red dots). As we can see the solutions coincide perfectly.

If $N_{f}=4$ then the level is at $N$ and the Yang-Mills term cancels. Hence this has the same behavior as we found for the $\mathrm{SU}(2 N)$ case. The eigenvalue distribution solving (6.19) is shown in figure 9(b). It shows a width equal to one and an exponential tail that can be approximated by $e^{\delta \sigma-1}$, as shown by the dashed blue line on the plot. Unfortunately, (6.24) is difficult to solve even numerically due to instabilities in the numerics.

Finally, if $N_{f}>4$ then the level is less than $N$ and there is a small negative YangMills contribution which still gives a stable eigenvalue distribution, as long as $N_{f}<8$. Interestingly, in this case the particular form of the distribution depends on whether $N_{f}$ is odd or even. In the case of even $N_{f}$ we get a picture similar to the $\mathrm{SU}(N)_{N}$ theory, i.e. an eigenvalue density with an exponential tail of the form $e^{\delta \sigma-1}$. An example of such a distribution is shown in figure 9 (c). In the case of odd $N_{f}$ there is no tail in the eigenvalue density and the support is finite of order one. The corresponding numerical solution to (6.19) is shown in figure $9(\mathrm{~d})$. It is not clear to us what causes the difference between even and odd $N_{f}$ and we leave this for future work. 


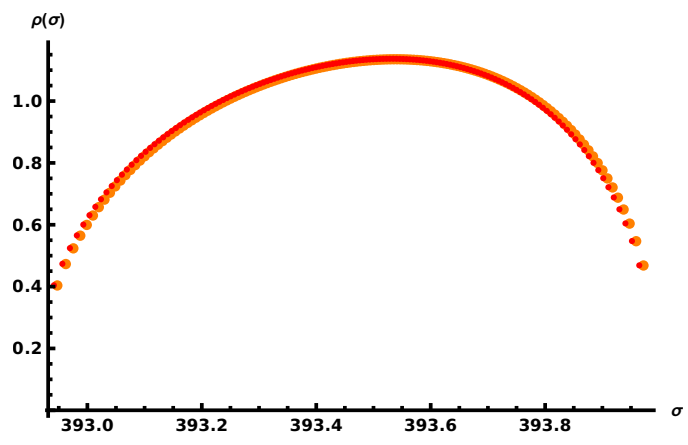

(a) Density for $N_{f}=2$

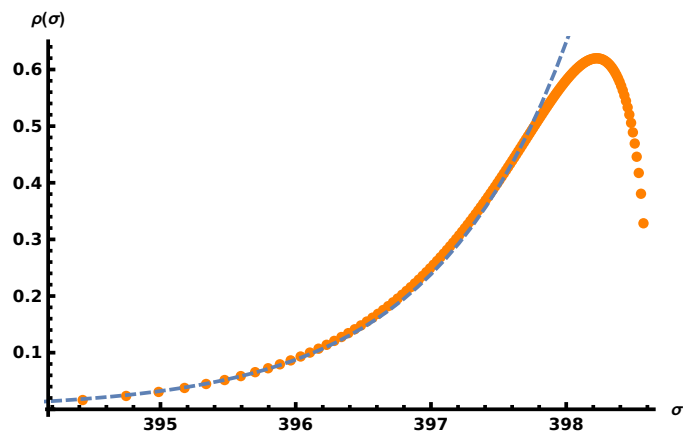

(c) Density for $N_{f}=6$

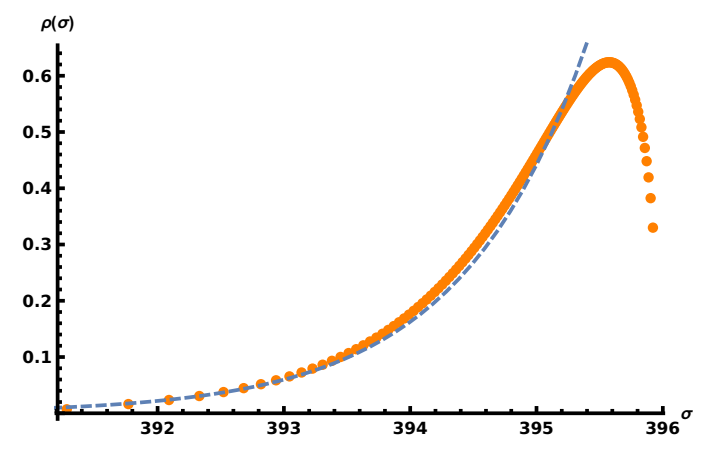

(b) Density for $N_{f}=4$

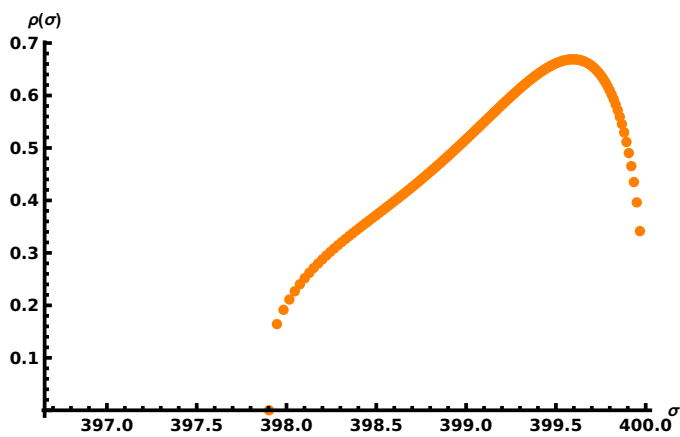

(d) Density for $N_{f}=7$

Figure 9. Eigenvalue density $\rho(\delta \sigma)$ for $\lambda_{\text {eff }}=-.1$ and $N=150$.

\subsection{An apparent fifth order phase transition and its possible resolution}

Let us now consider the behavior of the $\operatorname{USp}(2 N)$ gauge theory near the superconformal fixed point at $t \equiv \frac{8 \pi^{2}}{\lambda_{\text {eff }}}=0$. Previously we saw that the pure $\mathrm{SU}(N)$ gauge theory passes smoothly through the fixed point, while the $\mathrm{SU}(N / 2)_{N / 2}$ fixed point is a limiting value for the Yang-Mills coupling. In this case we will find something in between.

We consider once again the saddle-point equation (6.1), but this time with $\left|\lambda_{\text {eff }}\right| \gg 1$. At the fixed point the eigenvalues scale as $N^{1 / 2}$ [10]. In our case we expect the same behavior since we are only perturbing around the fixed point. Hence, the eigenvalues in general are widely separated in the large $N$ limit and we can simplify the saddle point equations (6.1) to

$$
\left(8-N_{f}\right) \sigma_{i}^{2}+2 N t \sigma_{i}=\frac{9}{4}(2 i-2),
$$

where we have assumed all eigenvalues are positive and ordered. This is just an algebraic equation for $\sigma_{i}$ with the solution

$$
\sigma_{i}=-\frac{N t}{\left(8-N_{f}\right)}+\frac{1}{2} \sqrt{\left(\frac{2 N t}{\left(8-N_{f}\right)}\right)^{2}+\frac{9(2 i-2)}{\left(8-N_{f}\right)}}
$$




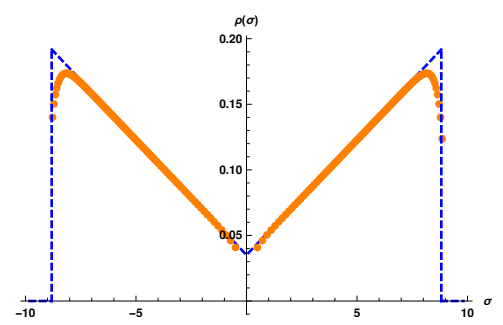

(a) $t=0.001 \times 8 \pi^{2}$

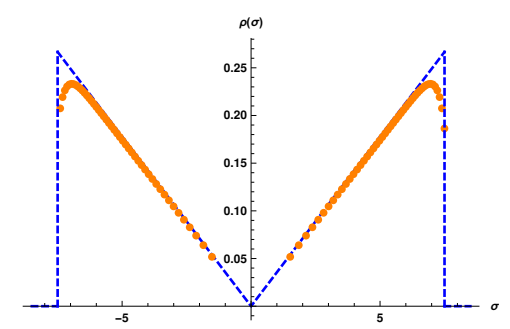

(b) $t=0$ (fixed point)

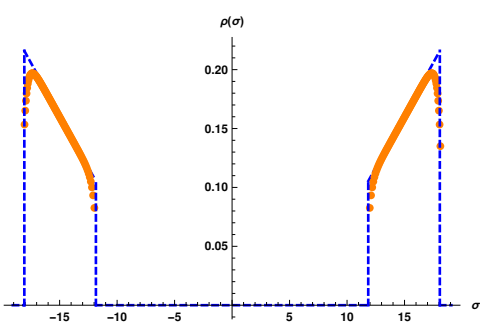

(c) $t=-0.003 \times 8 \pi^{2}$

Figure 10. Comparison of the analytical and numerical solutions in the vicinity of the fixed point $t=0$ for $N=100$ and $N_{f}=4$.

This in turn leads to the eigenvalue density

$$
\rho(\sigma)=\frac{4}{9} \frac{8-N_{f}}{N}\left(|\sigma|+\frac{2 N t}{\left(8-N_{f}\right)}\right)
$$

which is valid for both positive and negative coupling, but the endpoints are qualitatively different in the two cases. When $t>0$ the density has support between the points

$$
x_{1}^{+}=0, \quad x_{2}^{+}=-\frac{N t}{\left(8-N_{f}\right)}+\frac{1}{2} \sqrt{\left(\frac{2 N t}{\left(8-N_{f}\right)}\right)^{2}+\frac{18 N}{8-N_{f}}},
$$

while for $t<0$ the support is between

$$
x_{1}^{-}=-\frac{2 N t}{\left(8-N_{f}\right)}, \quad x_{2}^{-}=-\frac{N t}{\left(8-N_{f}\right)}+\frac{1}{2} \sqrt{\left(\frac{2 N t}{\left(8-N_{f}\right)}\right)^{2}+\frac{18 N}{8-N_{f}}} .
$$

In figure 10 we show the numerical solutions to the saddle point equation (6.1) and compare it with the analytical solutions (6.27), (6.28), (6.29) in the vicinity of the fixed point $t=0$. As we see there is an excellent match between the two and we can trust our approximations.

As is clear from figure 10, when passing through the fixed point the eigenvalue support splits into two parts. Usually this is attributed to a third order phase transition, which can be investigated by analyzing the free energy while crossing the fixed point. In the present case the free energy is approximated by

$$
\begin{aligned}
F= & \pi N^{2} t \int d \sigma \sigma^{2} \rho(\sigma)+\frac{\pi N\left(8-N_{f}\right)}{3} \int d \sigma \sigma^{3} \rho(\sigma) \\
& -\frac{9 \pi}{8} N^{2} \int \delta \sigma \rho(\sigma) \int \delta \sigma^{\prime} \rho\left(\sigma^{\prime}\right)\left(\left|\sigma-\sigma^{\prime}\right|+\left|\sigma+\sigma^{\prime}\right|\right),
\end{aligned}
$$

where the integrals are evaluated between the endpoints of the support given in (6.28) and (6.29) for positive and negative $t$ respectively.

The calculation of these integrals is straightforward but results in large and not particularly informative expressions. Instead we wish to know the order of the discontinuity in the derivatives of the free energy with respect to $t$ at $t=0$. This task can be simplified by 


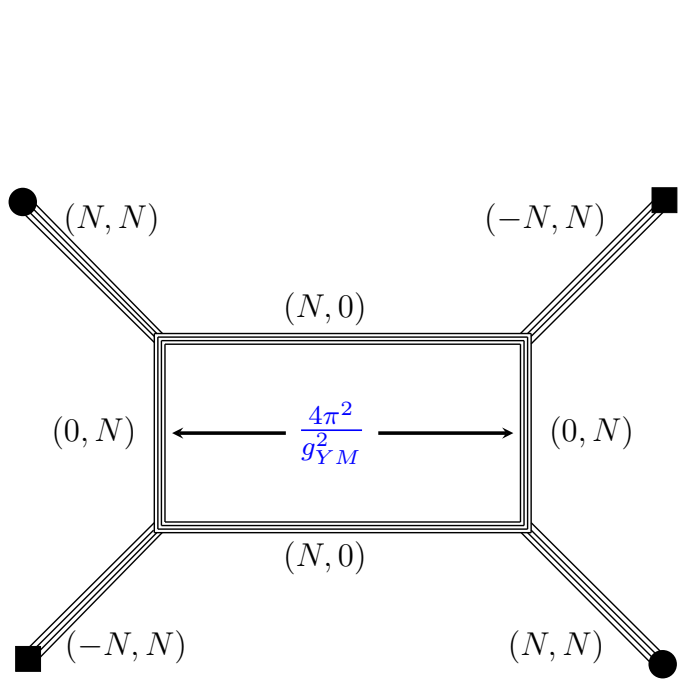

(a) $g_{\mathrm{YM}}^{2}>0$

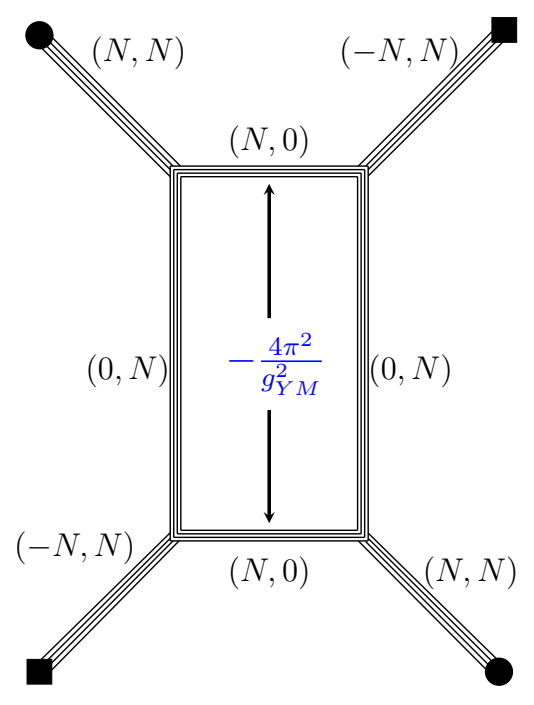

(b) $g_{\mathrm{YM}}^{2}<0$

Figure 11. $(p, q)$ web for a $\operatorname{USp}(2 N)$ gauge theory with a hypermultiplet in the antisymmetric representation at positive and negative coupling.

using the relation between the first derivative of the free energy with the second moment of the eigenvalue density, namely

$$
\left\langle\sigma^{2}\right\rangle=\frac{1}{\pi N^{2}} \frac{\partial}{\partial t} F=\int d \sigma \sigma^{2} \rho(\sigma) .
$$

After a straightforward computation one then finds that

$$
\left.F\right|_{t \rightarrow 0^{+}}-\left.F\right|_{t \rightarrow 0^{-}}=\frac{16 \pi N^{5}}{135} t^{5}+\mathrm{O}\left(t^{9}\right)
$$

Hence, this system seems to have a fifth order phase transition at the superconformal fixed point.

But this result contradicts the expected self-duality for a $\operatorname{USp}(2 N)$ gauge theory with an antisymmetric hypermultiplet under the change of sign of the coupling, $\lambda \rightarrow-\lambda$. One way to see the duality is to observe that in the UV this theory flows to the rank $N E_{1}$ theory which has an $\mathrm{SU}(2)_{g} \times \mathrm{SU}(2)_{m}$ global symmetry. The $\mathrm{SU}(2)_{m}$ flips the sign of the mass term, which in our case emerges even at the level of the saddle point equations (6.1). The $\mathrm{SU}(2)_{g}$ is the symmetry responsible for flipping the sign of the YM coupling $g_{\mathrm{YM}}^{2} \rightarrow-g_{\mathrm{YM}}^{2}$. The existence of this global symmetry at the fixed point should lead to the self-duality of the $\operatorname{USp}(2 N)$ theory. The $(p, q)$ brane web construction for the theory [29], shown in figure 11, makes the self-duality immediately obvious. This brane web is a direct higher rank generalization of the $\mathrm{SU}(2)$ brane web shown in figure 1. As we can see the brane web is self-dual under the exchange of $p$ and $q$, reflecting the duality between negative and positive coupling.

The most likely resolution for the contradiction is through the inclusion of the instantons. In section 5 we have analyzed the instanton contribution to the partition function 
for $\mathrm{SU}(N) \mathrm{SYM}$ and showed that it can be neglected in the large $N$ limit. However, a similar analysis in the case of $\operatorname{USp}(2 N)$ is more complicated due to the hypermultiplet in the antisymmetric representation. In the case of $\mathrm{SU}(2)$, instantons are crucial for the establishment of the duality. We expect the same for $\operatorname{USp}(2 N)$ with the antisymmetric hypermultiplet. In this case the contribution of the instantons will wash out the fifth order phase transition and turn it to a smooth crossover [30].

\section{Discussion and outlook}

In this paper we considered five-dimensional supersymmetric gauge theories in the large $N$ limit with negative gauge coupling. In particular we considered two important types of theories, pure $\mathcal{N}=1 \mathrm{SU}(N)$ super Yang-Mills and a $\operatorname{USp}(2 N)$ theory with an antisymmetric and $N_{f}<8$ fundamental hypermultiplets. Using supersymmetric localization we showed how the eigenvalues of the resulting matrix model separate into blocks of order one as we cross over to negative gauge coupling through the infinite coupling fixed point. This is consistent with the $\mathrm{SU}(N)$ theory breaking to $\mathrm{SU}(N / 2)_{+N / 2} \times \mathrm{SU}(N / 2)_{-N / 2} \times \mathrm{SU}(2)$ for the

case of $N$ even. For $N$ odd we find the group breaking to $\mathrm{SU}\left(\frac{N-1}{2}\right)_{+\frac{N+1}{2}} \times \mathrm{SU}\left(\frac{N-1}{2}\right)_{-\frac{N+1}{2}} \times$ $\mathrm{U}(1)^{2}$, where we expect the $\mathrm{U}(1)^{2}$ to be enhanced to $\mathrm{SU}(2)^{2}$. A similar analysis for the $\mathrm{USp}(2 N)$ theory with the antisymmetric tensor shows that after passing through the infinite coupling fixed point the gauge group breaks to the $\mathrm{SU}(N)_{8-N_{f}} \times \mathrm{SU}(N)_{N_{f}-8}$ undergoing an apparent fifth order phase transition which we conjecture to be smoothed out by instanton contributions in order to restore the self-duality.

Although we have focused only on these two cases, the pattern we observed seems generic for large $N$ gauge theories. In the future it would be interesting to generalize this analysis to other theories admitting infinite coupling fixed points. There are two classes of particular interest. The first class is a family of quiver theories with massive type IIA $\mathrm{AdS}_{6}$ duals proposed in [12]. The $S^{5}$ partition functions of these theories were analyzed in [10]. The second class consists of quiver theories with type IIB $\mathrm{AdS}_{6}$ duals [31-33]. The large $N$ limit of these theories was analyzed recently using localization in [34]. Since all of these quivers admit a large $N$ limit we can expect that our analysis can be applied to them. Because these theories possess multiple gauge nodes one can expect some interesting peculiarities.

Recently, the complete prepotential of certain five-dimensional low rank gauge theories was proposed which is valid in the entire region of parameter space [35]. It would be interesting to extend the complete prepotential to arbitrarily high rank theories and compare this with the localization results described in this paper.

\section{Acknowledgments}

We thank O. Bergman, J. Qiu and M. Zabzine for helpful discussions. We also thank O. Bergman for important comments about the first version of this paper. The research of J.A.M. is supported in part by Vetenskapsrådet under grant \#2016-03503 and by the Knut and Alice Wallenberg Foundation under grant Dnr KAW 2015.0083. JAM thanks the 
Center for Theoretical Physics at MIT for kind hospitality during the course of this work. The research of A.N. is supported by Israel Science Foundation under grant No. 2289/18, and by the I-CORE Program of the Planning and Budgeting Committee

\section{A Width of the eigenvalue distribution at finite $N$}

Consider the generalization of the eigenvalue equation in (3.10)

$$
M\left(\sigma_{i}^{2}+\chi\right)=\sum_{j \neq i}^{M}\left(2 q-\left(\sigma_{i}-\sigma_{j}\right)^{2}\right) \operatorname{coth}\left(\pi\left(\sigma_{i}-\sigma_{j}\right)\right),
$$

where we have replaced $N / 2$ with $M$ and we assume that $\sum_{i} \sigma_{i}=0$. One can easily show that $\chi=-\frac{1}{M} \sum_{i} \sigma_{i}^{2}$ by summing over $i$ and noticing that the right hand side is zero because of the antisymmetry between $\sigma_{i}$ and $\sigma_{j}$. We then wish to show that $M \chi=-(M-1) q$. Let us assume that this is true, in which case the left hand side of (A.1) becomes

$$
M\left(\sigma_{i}^{2}+\chi\right)=-\sum_{j \neq i}\left(2 q-\left(\sigma_{i}-\sigma_{j}\right)^{2}\right),
$$

and (A.1) can then be rewritten as

$$
0=\sum_{j \neq i}^{M}\left(2 q-\left(\sigma_{i}-\sigma_{j}\right)^{2}\right)\left(\operatorname{coth}\left(\pi\left(\sigma_{i}-\sigma_{j}\right)\right)+1\right)
$$

for all $i$. The $i=M$ equation in (A.3) follows from the other $M-1$ equations.

To prove the claim we then need to show that the $i=M-1$ equation in (A.3) follows from the first $M-2$ equations. To this end we note that we can write

$$
2 q-\left(\sigma_{M-1}-\sigma_{M}\right)^{2}=-\sum_{i<j}^{M}\left(2 q-\left(\sigma_{i}-\sigma_{j}\right)^{2}\right)+\left(2 q-\left(\sigma_{M-1}-\sigma_{M}\right)^{2}\right)
$$

If we then use that

$$
\operatorname{coth}\left(\pi\left(\sigma_{M-1}-\sigma_{M}\right)\right)=\frac{\operatorname{coth}\left(\pi\left(\sigma_{i}-\sigma_{M-1}\right) \operatorname{coth}\left(\pi\left(\sigma_{i}-\sigma_{M}\right)\right)-1\right.}{\operatorname{coth}\left(\pi\left(\sigma_{i}-\sigma_{M-1}\right)-\operatorname{coth}\left(\pi\left(\sigma_{i}-\sigma_{M}\right)\right)\right.}
$$

for any $i$, then using the first $M-2$ equations, the right hand side of the $i=M-1$ equation can be rewritten as

$$
\begin{aligned}
& \sum_{i<j}^{M-2}\left(2 q-\left(\sigma_{i}-\sigma_{j}\right)^{2}\right)\left[\frac{\left(\operatorname{coth}\left(\pi\left(\sigma_{i}-\sigma_{M-1}\right)\right)-1\right)\left(\operatorname{coth}\left(\pi\left(\sigma_{i}-\sigma_{j}\right)\right)+1\right)}{\operatorname{coth}\left(\pi\left(\sigma_{i}-\sigma_{M-1}\right)\right)-\operatorname{coth}\left(\pi\left(\sigma_{i}-\sigma_{M}\right)\right)}\right. \\
& \left.\quad+\frac{\left(\operatorname{coth}\left(\pi\left(\sigma_{j}-\sigma_{M-1}\right)\right)-1\right)\left(\operatorname{coth}\left(\pi\left(\sigma_{j}-\sigma_{i}\right)\right)+1\right)}{\operatorname{coth}\left(\pi\left(\sigma_{j}-\sigma_{M-1}\right)\right)-\operatorname{coth}\left(\pi\left(\sigma_{j}-\sigma_{M}\right)\right)}-\operatorname{coth}\left(\pi\left(\sigma_{M-1}-\sigma_{M}\right)\right)-1\right] .
\end{aligned}
$$

The term inside the square brackets is zero for each $i$ and $j$, hence proving that the width squared of the eigenvalue distribution is given by

$$
\frac{1}{M} \sum_{i} \sigma_{i}^{2}=\frac{M-1}{M} q
$$




\section{B Exponential behavior for instantons}

We can derive the general behavior in (5.6) as follows. Suppose we start with a large value of $N$, say $N=2 M_{0} \gg 1$, and call the product in (5.6) $P\left(M_{0}\right)$. Now double the value of $N$ and assume that the new points lie on top of the original ones and also halfway between the originals. Let us also assume that $i$ is at the maximum and we will also use half-integer numbering for the $j$ index. Then for a typical distribution we would replace $\sinh ^{2}\left(\pi\left(\delta_{j i}\right)\right)$ with

$$
\begin{aligned}
& \sinh \left(\pi\left(\delta \sigma_{j-1 / 2}-\delta \sigma_{i}\right)\right) \sinh ^{2}\left(\pi\left(\delta \sigma_{j i}\right)\right) \sinh \left(\pi\left(\delta \sigma_{j+1 / 2}-\delta \sigma_{i}\right)\right) \\
& \approx \sinh ^{4}\left(\pi\left(\delta \sigma_{j i}\right)\right)\left(1-\frac{\pi^{2}}{16 M_{0}^{2} \rho^{2}\left(\delta \sigma_{j}\right) \sinh ^{2}\left(\pi\left(\delta \sigma_{j i}\right)\right)}\right)
\end{aligned}
$$

where we used that $\delta \sigma_{j}-\delta \sigma_{j-1 / 2} \approx \frac{1}{4 M_{0} \rho\left(\delta \sigma_{j}\right)}$. For $\left|\delta \sigma_{j}-\delta \sigma_{i}\right| \ll 1$ we can approximate the term inside the parentheses in (B.1) as $1-\frac{1}{4(i-j)^{2}}$ and it rapidly converges toward 1 as $j$ moves away from $i$. The product of all such terms is then well approximated by $4 / \pi^{2}$. However, in our doubling we have not yet accounted for the term

$$
\sinh \left(\pi\left(\delta \sigma_{i-1 / 2}-\delta \sigma_{i}\right)\right) \sinh \left(\pi\left(\delta \sigma_{i+1 / 2}-\delta \sigma_{i}\right)\right)\left(\frac{\sinh \left(\pi\left(\delta \sigma_{1}-\delta \sigma_{i}\right)\right)}{\sinh \left(\pi\left(\delta \sigma_{M_{0}}-\delta \sigma_{i}\right)\right)}\right)^{ \pm 1} \approx \frac{k}{M_{0}^{2}}
$$

where $k$ is a positive constant and where the choice of sign depends on whether we place a leftover point to the left or the right of the distribution. Putting this all together we find that

$$
P\left(2 M_{0}\right)=C M_{0}^{2} P\left(M_{0}\right)^{2},
$$

where $C$ is a constant. Now we can do the process over again, where by following the same logic we have

$$
P\left(4 M_{0}\right)=C\left(2 M_{0}\right)^{2} P\left(2 M_{0}\right)^{2}=4 C^{3} M_{0}^{6} P\left(M_{0}\right)^{4} .
$$

It is then straightforward to show that

$$
P\left(2^{m} M_{0}\right)=\frac{1}{4 C M_{0}^{2}}\left(4 C M_{0}^{2} P\left(M_{0}\right)\right)^{2^{m}} 2^{-2 m} .
$$

If we now let $N=2 \cdot 2^{m} M_{0}$ we can express the product as

$$
P(N / 2)=\frac{1}{C N^{2}}\left[\left(4 C M_{0}^{2} P\left(M_{0}\right)\right)^{\frac{1}{2 M_{0}}}\right]^{N},
$$

showing the general form in (5.6). If the term inside the square brackets is less than 1 then there is exponential suppression.

Open Access. This article is distributed under the terms of the Creative Commons Attribution License (CC-BY 4.0), which permits any use, distribution and reproduction in any medium, provided the original author(s) and source are credited. 


\section{References}

[1] N. Seiberg, Five-dimensional SUSY field theories, nontrivial fixed points and string dynamics, Phys. Lett. B 388 (1996) 753 [hep-th/9608111] [INSPIRE].

[2] O. Aharony and A. Hanany, Branes, superpotentials and superconformal fixed points, Nucl. Phys. B 504 (1997) 239 [hep-th/9704170] [INSPIRE].

[3] O. Aharony, A. Hanany and B. Kol, Webs of $(p, q)$ five-branes, five-dimensional field theories and grid diagrams, JHEP 01 (1998) 002 [hep-th/9710116] [INSPIRE].

[4] V. Pestun, Localization of gauge theory on a four-sphere and supersymmetric Wilson loops, Commun. Math. Phys. 313 (2012) 71 [arXiv:0712.2824] [INSPIRE].

[5] V. Pestun et al., Localization techniques in quantum field theories, J. Phys. A 50 (2017) 440301 [arXiv: 1608.02952] [INSPIRE].

[6] J.G. Russo and K. Zarembo, Large $N$ Limit of $\mathcal{N}=2 \mathrm{SU}(N)$ Gauge Theories from Localization, JHEP 10 (2012) 082 [arXiv:1207.3806] [INSPIRE].

[7] G. Lockhart and C. Vafa, Superconformal Partition Functions and Non-perturbative Topological Strings, JHEP 10 (2018) 051 [arXiv:1210.5909] [INSPIRE].

[8] J. Qiu and M. Zabzine, Factorization of $5 D$ super Yang-Mills theory on $Y^{p, q}$ spaces, Phys. Rev. D 89 (2014) 065040 [arXiv:1312.3475] [INSPIRE].

[9] J. Qiu, L. Tizzano, J. Winding and M. Zabzine, Gluing Nekrasov partition functions, Commun. Math. Phys. 337 (2015) 785 [arXiv:1403.2945] [InSPIRE].

[10] D.L. Jafferis and S.S. Pufu, Exact results for five-dimensional superconformal field theories with gravity duals, JHEP 05 (2014) 032 [arXiv: 1207.4359] [INSPIRE].

[11] A. Brandhuber and Y. Oz, The D4-D8 brane system and five-dimensional fixed points, Phys. Lett. B 460 (1999) 307 [hep-th/9905148] [INSPIRE].

[12] O. Bergman and D. Rodriguez-Gomez, 5d quivers and their AdS 6 duals, JHEP 07 (2012) 171 [arXiv: 1206.3503] [INSPIRE].

[13] J.A. Minahan, Localizing gauge theories on $S^{d}$, JHEP 04 (2016) 152 [arXiv:1512.06924] [INSPIRE].

[14] A. Gorantis, J.A. Minahan and U. Naseer, Analytic continuation of dimensions in supersymmetric localization, JHEP 02 (2018) 070 [arXiv:1711.05669] [INSPIRE].

[15] J. Källén and M. Zabzine, Twisted supersymmetric 5D Yang-Mills theory and contact geometry, JHEP 05 (2012) 125 [arXiv:1202.1956] [INSPIRE].

[16] J. Källén, J. Qiu and M. Zabzine, The perturbative partition function of supersymmetric $5 D$ Yang-Mills theory with matter on the five-sphere, JHEP 08 (2012) 157 [arXiv:1206.6008] [INSPIRE].

[17] J. Qiu and M. Zabzine, Review of localization for $5 d$ supersymmetric gauge theories, J. Phys. A 50 (2017) 443014 [arXiv: 1608.02966] [INSPIRE].

[18] N. Bobev, P. Bomans, F.F. Gautason, J.A. Minahan and A. Nedelin, Supersymmetric Yang-Mills, Spherical Branes, and Precision Holography, JHEP 03 (2020) 047 [arXiv: 1910.08555] [INSPIRE].

[19] J.A. Minahan, A. Nedelin and M. Zabzine, 5D super Yang-Mills theory and the correspondence to $A d S_{7} / C F T_{6}$, J. Phys. A 46 (2013) 355401 [arXiv:1304.1016] [INSPIRE]. 
[20] A. Nedelin, Phase transitions in 5D super Yang-Mills theory, JHEP 07 (2015) 004 [arXiv: 1502.07275] [INSPIRE].

[21] K.A. Intriligator, D.R. Morrison and N. Seiberg, Five-dimensional supersymmetric gauge theories and degenerations of Calabi-Yau spaces, Nucl. Phys. B 497 (1997) 56 [hep-th/9702198] [INSPIRE].

[22] O. Bergman and D. Rodríguez-Gómez, The Cat's Cradle: Deforming the higher rank $E_{1}$ and $\tilde{E}_{1}$ theories, arXiv:2011.05125 [INSPIRE].

[23] K. Hosomichi, R.-K. Seong and S. Terashima, Supersymmetric Gauge Theories on the Five-Sphere, Nucl. Phys. B $\mathbf{8 6 5}$ (2012) 376 [arXiv:1203.0371] [INSPIRE].

[24] H.-C. Kim and S. Kim, M5-branes from gauge theories on the 5-sphere, JHEP 05 (2013) 144 [arXiv: 1206.6339] [INSPIRE].

[25] E. Witten, Phase transitions in M-theory and F-theory, Nucl. Phys. B 471 (1996) 195 [hep-th/9603150] [INSPIRE].

[26] T. Okuda and V. Pestun, On the instantons and the hypermultiplet mass of $N=2^{*}$ super Yang-Mills on $S^{4}$, JHEP 03 (2012) 017 [arXiv: 1004.1222] [INSPIRE].

[27] J. Kallen, J.A. Minahan, A. Nedelin and M. Zabzine, $N^{3}$-behavior from $5 D$ Yang-Mills theory, JHEP 10 (2012) 184 [arXiv:1207.3763] [INSPIRE].

[28] J.A. Minahan and A. Nedelin, Phases of planar 5-dimensional supersymmetric Chern-Simons theory, JHEP 12 (2014) 049 [arXiv: 1408.2767] [INSPIRE].

[29] O. Bergman and G. Zafrir, 5d fixed points from brane webs and O7-planes, JHEP 12 (2015) 163 [arXiv: 1507.03860] [INSPIRE].

[30] J.A. Minahan and A. Nedelin, Instanton contributions in five-dimensional $\mathcal{N}=1$ theories, in progress.

[31] E. D'Hoker, M. Gutperle, A. Karch and C.F. Uhlemann, Warped $A d S_{6} \times S^{2}$ in Type IIB supergravity I: Local solutions, JHEP 08 (2016) 046 [arXiv: 1606. 01254] [INSPIRE].

[32] E. D'Hoker, M. Gutperle and C.F. Uhlemann, Holographic duals for five-dimensional superconformal quantum field theories, Phys. Rev. Lett. 118 (2017) 101601 [arXiv: 1611.09411] [INSPIRE].

[33] E. D'Hoker, M. Gutperle and C.F. Uhlemann, Warped $A d S_{6} \times S^{2}$ in Type IIB supergravity II: Global solutions and five-brane webs, JHEP 05 (2017) 131 [arXiv:1703.08186] [INSPIRE].

[34] C.F. Uhlemann, Exact results for 5d SCFTs of long quiver type, JHEP 11 (2019) 072 [arXiv: 1909.01369] [INSPIRE].

[35] H. Hayashi, S.-S. Kim, K. Lee and F. Yagi, Complete prepotential for $5 d \mathcal{N}=1$ superconformal field theories, JHEP 02 (2020) 074 [arXiv:1912.10301] [INSPIRE]. 